\title{
RENORMALIZATION PROPERTIES OF SOFTLY BROKEN SUSY GAUGE THEORIES円
}

\author{
Dmitri KAZAKOV \\ Bogoliubov Laboratory of Theoretical Physics, Joint Institute for Nuclear Research, \\ Dubna, Russia \\ and \\ Institute for Theoretical and Experimental Physics, Moscow, Russia
}

\begin{abstract}
In the present review we show that renormalizations in a softly broken SUSY gauge theory are not independent but directly follow from those of an unbroken or rigid theory. This is a consequence of a treatment of a softly broken theory as a rigid one in external spurion superfield. This enables one to get the singular part of effective action in a broken theory from a rigid one by a simple modification of the couplings. Substituting the modified couplings into renormalization constants, RG equations, solutions to these equations, approximate solutions, fixed points, etc., one can get corresponding relations for the soft terms by a simple Taylor expansion over the Grassmannian variables. Some examples including the MSSM in low and high $\tan \beta$ regime, SUSY GUTs and the $\mathrm{N}=2$ Seiberg-Witten model are considered.
\end{abstract}

\section{Introduction}

In a series of papers [1] $]^{-}[$[5] we have shown that renormalizations in a softly broken SUSY theory follow from those of an unbroken one in a straightforward way. This is in agreement with the other approaches [6, 7, 8] and is inspired by the original observation of Ref.[9]. In what follows we give a review of our approach. It does not explicitly use the power of holomorphicity advocated by some authors [7, 8], but ends up with the simple and straightforward algorithm which is easy to apply.

The main idea is that a softly broken supersymmetric gauge theory can be considered as a rigid SUSY theory imbedded into external space-time independent superfield $\eta$, so that all couplings and masses become external superfields $S(\eta, \bar{\eta})$. Then, the following crucial statement is valid [1]

The statement: In external spurion field $\eta$ the $U V$ singular part of the effective action depends on the couplings $S(\eta, \bar{\eta})$, but does not depend on their derivatives:

$$
S_{\text {Sing }}^{e f f}(g) \Rightarrow S_{\text {Sing }}^{e f f}\left(S, D^{2} / S, \bar{D}^{2} / S, D^{2} \bar{D}^{2} S\right)
$$

where $D$ and $\bar{D}$ are the supercovariant derivatives, and as a result has the same form in unbroken and broken cases.

\footnotetext{
${ }^{1}$ Talk presented at the conference "Continuous Advances in QCD 2002/Arkadyfest", Minnesota, May 2002 .
} 
With replacement of the couplings by external fields one can calculate the effective action $S_{\text {Sing }}^{e f f}(g)$ assuming that the external field is a constant, i.e. in a rigid theory. This approach to a softly broken supersymmetric theory allows us to use remarkable mathematical properties of $N=1$ SUSY field theories such holomorphicity which leads to the non-renormalization theorems, cancellation of quadratic divergences, etc.

The renormalization procedure in a softly broken SUSY gauge theory can be performed in the following way:

One takes the renormalization constants of a rigid theory, calculated in some massless scheme, substitutes instead of the rigid couplings (gauge and Yukawa) their modified expressions, which depend on a Grassmannian variable, and expand over this variable. This gives renormalization constants for the soft terms. Differentiating them with respect to a scale one can find corresponding renormalization group equations.

Thus, the soft-term renormalizations are not independent but can be calculated from the known renormalizations of a rigid theory with the help of the differential operators. Explicit form of these operators has been found in a general case and in some particular models like SUSY GUTs or the MSSM [1, 3]. The same expressions have been obtained also in a somewhat different approach in Ref. [6, 7, 10].

In fact as it has been shown in [3] this procedure works at all stages. One can make the above mentioned substitution on the level of the renormalization constants, RG equations, solutions to these equations, approximate solutions, fixed points, finiteness conditions, etc. Expanding then over a Grassmannian variable one obtains corresponding expressions for the soft terms. This way one can get new solutions of the RG equations and explore their asymptotics, or approximate solutions, or find their stability properties, starting from the known expressions for a rigid theory.

Throughout the paper we assume the existence of some gauge and SUSY invariant regularization. Though it is some problem by itself, in principle it is solvable [11]. Provided the rigid theory is well defined, we consider the modifications which appear due to the presence of soft SUSY breaking terms. To be more precise, when discussing one, two and three loop calculations of the renormalization constants we have in mind dimensional reduction and the minimal subtraction scheme. Though dimensional reduction is not selfconsistent in general [12], it is safe to use it in low orders and all the actual calculations are performed in the framework of dimensional reduction [13, 14, 15, 16]. Nevertheless, our main formulae have general validity provided the invariant procedure exists.

Below we give some examples: the general SUSY gauge theory in higher loops, the MSSM in low $\tan \beta$ regime where analytical solutions to the one-loop RG equations are known exactly and in high $\tan \beta$ regime where analytical solutions are known in iterative or approximate form. We discuss some particular solutions like the fixed point ones and examine their properties. The method allows one to get the same type of solutions for the soft SUSY breaking terms. The other examples are the finite $\mathrm{N}=1 \mathrm{SUSY}$ GUTs and the $\mathrm{N}=2$ Seiberg-Witten model where exact (nonperturbative) solution is known. Here one can extend finiteness and the S-W solution to the soft terms as well. 


\section{Soft SUSY Breaking and the Spurion Superfields}

Consider an arbitrary $N=1$ SUSY gauge theory with unbroken SUSY within the superfield formalism. The Lagrangian of a rigid theory is given by

$$
\begin{aligned}
\mathcal{L}_{\text {rigid }} & =\int d^{2} \theta \frac{1}{4 g^{2}} \operatorname{Tr} W^{\alpha} W_{\alpha}+\int d^{2} \bar{\theta} \frac{1}{4 g^{2}} \operatorname{Tr} \bar{W}_{\dot{\alpha}} \bar{W}^{\dot{\alpha}} \\
& +\int d^{2} \theta d^{2} \bar{\theta} \bar{\Phi}^{i}\left(e^{V}\right)_{i}^{j} \Phi_{j}+\int d^{2} \theta \mathcal{W}+\int d^{2} \bar{\theta} \quad \overline{\mathcal{W}}
\end{aligned}
$$

where

$$
W_{\alpha}=-\frac{1}{4} \bar{D}^{2} e^{-V} D_{\alpha} e^{V}, \quad \bar{W}_{\dot{\alpha}}=-\frac{1}{4} D^{2} e^{-V} \bar{D}_{\dot{\alpha}} e^{V},
$$

are the gauge field strength tensors and the superpotential $\mathcal{W}$ has the form

$$
\mathcal{W}=\frac{1}{6} y^{i j k} \Phi_{i} \Phi_{j} \Phi_{k}+\frac{1}{2} M^{i j} \Phi_{i} \Phi_{j}
$$

To fix the gauge, the usual gauge-fixing term can be introduced. It is useful to choose it in the form

$$
\mathcal{L}_{\text {gauge-fixing }}=-\frac{1}{16} \int d^{2} \theta d^{2} \bar{\theta} \operatorname{Tr}(\bar{f} f+f \bar{f})
$$

where the gauge fixing condition is taken as

$$
f=\bar{D}^{2} \frac{V}{\sqrt{\xi g^{2}}}, \quad \bar{f}=D^{2} \frac{V}{\sqrt{\xi g^{2}}} .
$$

Here $\xi$ is the usual gauge-fixing parameter. Then, the corresponding ghost term is [17]

$$
\mathcal{L}_{\text {ghost }}=i \int d^{2} \theta \frac{1}{4} \operatorname{Tr} b \delta_{c} f-i \int d^{2} \bar{\theta} \frac{1}{4} \operatorname{Tr} \bar{b} \delta_{\bar{c}} \bar{f}
$$

where $c$ and $b$ are the Faddeev-Popov ghost and antighost chiral superfields, respectively, and $\delta_{c}$ is the gauge transformation with the replacement of gauge superfield parameters $\Lambda(\bar{\Lambda})$ by chiral (antichiral) ghost fields $c(\bar{c})$.

For our choice of the gauge-fixing condition, the gauge transformation of $f$ looks like

$$
\delta_{\Lambda} f=\bar{D}^{2} \delta_{\Lambda} \frac{V}{\sqrt{\xi g^{2}}}=i \bar{D}^{2} \frac{1}{\sqrt{\xi g^{2}}} \mathcal{L}_{V / 2}\left[\Lambda+\bar{\Lambda}+\operatorname{coth}\left(\mathcal{L}_{V / 2}\right)(\Lambda-\bar{\Lambda})\right]
$$

where $\mathcal{L}_{X} Y \equiv[X, Y]$. Equation (6) then takes the form

$$
\begin{gathered}
\mathcal{L}_{\text {ghost }}=-\int d^{2} \theta \frac{1}{4} \operatorname{Tr} b \bar{D}^{2} \frac{1}{\sqrt{\xi g^{2}}} \mathcal{L}_{V / 2}\left[c+\bar{c}+\operatorname{coth}\left(\mathcal{L}_{V / 2}\right)(c-\bar{c})\right]+\text { h.c. } \\
=\int d^{2} \theta d^{2} \bar{\theta} \operatorname{Tr}\left(\frac{b+\bar{b}}{\sqrt{\xi g^{2}}}\right) \mathcal{L}_{V / 2}\left[c+\bar{c}+\operatorname{coth}\left(\mathcal{L}_{V / 2}\right)(c-\bar{c})\right] \\
=\int d^{4} \theta \operatorname{Tr}\left(\frac{b+\bar{b}}{\sqrt{\xi g^{2}}}\right)\left((c-\bar{c})+\frac{1}{2}[V,(c+\bar{c})]+\frac{1}{12}[V,[V,(c-\bar{c})]]+\ldots\right) .
\end{gathered}
$$


The resulting Lagrangian together with the gauge-fixing and the ghost terms are invariant under the BRST transformations. For a rigid theory in our normalization of the fields, they have the form 17

$$
\begin{aligned}
& \delta V=\epsilon \mathcal{L}_{V / 2}\left[c+\bar{c}+\operatorname{coth}\left(\mathcal{L}_{V / 2}\right)(c-\bar{c})\right], \\
& \delta c^{a}=-\frac{i}{2} \epsilon f^{a b c} c^{b} c^{c}, \quad \delta \bar{c}^{a}=-\frac{i}{2} \epsilon f^{a b c} \bar{c}^{b} \bar{c}^{c}, \\
& \delta b^{a}=\frac{1}{8} \epsilon \bar{D}^{2} \bar{f}^{a}, \quad \delta \bar{b}^{a}=\frac{1}{8} \epsilon D^{2} f^{a} .
\end{aligned}
$$

Breaking of supersymmetry is the problem by itself. We do not discuss here the origin of SUSY breaking but rather concentrate on the consequences of it. Usually one considers the so-called "soft" breaking of SUSY, which means that the breaking terms do not spoil renormalizability of the theory and, in particular, the cancellation of quadratic divergences and are represented by the operators of dimension less than 4 [18]. Hence, to perform the SUSY breaking, that satisfies the requirement of "softness", one can introduce a gaugino mass term as well as cubic and quadratic interactions of scalar superpartners of the matter fields

$$
-\mathcal{L}_{\text {soft }-b r}=\left[\frac{M}{2} \lambda \lambda+\frac{1}{6} A^{i j k} \phi_{i} \phi_{j} \phi_{k}+\frac{1}{2} B^{i j} \phi_{i} \phi_{j}+\text { h.c. }\right]+\left(m^{2}\right)_{j}^{i} \phi_{i}^{*} \phi^{j},
$$

where $\lambda$ is the gaugino field, and $\phi_{i}$ is the lowest component of the chiral matter superfield.

This is not the most general form of the soft terms. In principle, one can add the terms like $\bar{\psi} \psi, \phi^{*} \phi \phi$, etc. 19]. However, the conventional choice (10) is sufficient for the goal of SUSY breaking and in what follows we stick to it.

Remarkably, one can rewrite the Lagrangian (10) in terms of $\mathrm{N}=1$ superfields introducing the external spurion superfields [18] $\eta=\theta^{2}$ and $\bar{\eta}=\bar{\theta}^{2}$, where $\theta$ and $\bar{\theta}$ are the Grassmannian parameters, as [9]

$$
\begin{aligned}
\mathcal{L}_{\text {soft }}= & \int d^{2} \theta \frac{1}{4 g^{2}}\left(1-2 M \theta^{2}\right) \operatorname{Tr} W^{\alpha} W_{\alpha}+\int d^{2} \bar{\theta} \frac{1}{4 g^{2}}\left(1-2 \bar{M} \bar{\theta}^{2}\right) \operatorname{Tr} \bar{W}^{\dot{\alpha}} \bar{W}_{\dot{\alpha}} \\
& +\int d^{2} \theta d^{2} \bar{\theta} \bar{\Phi}^{i}\left(\delta_{i}^{k}-\left(m^{2}\right)_{i}^{k} \eta \bar{\eta}\right)\left(e^{V}\right)_{k}^{j} \Phi_{j} \\
& +\int d^{2} \theta\left[\frac{1}{6}\left(y^{i j k}-A^{i j k} \eta\right) \Phi_{i} \Phi_{j} \Phi_{k}+\frac{1}{2}\left(M^{i j}-B^{i j} \eta\right) \Phi_{i} \Phi_{j}\right]+\text { h.c. }
\end{aligned}
$$

Thus, one can interpret the soft terms as the modification of the couplings of a rigid theory. The couplings become external superfields depending on Grassmannian parameters $\theta$ and $\bar{\theta}$. To get the explicit expression for the modified couplings, consider eqs.(11). The first two terms give [1]

$$
\frac{1}{g^{2}} \rightarrow \frac{1}{\tilde{g}^{2}}=\frac{1-M \theta^{2}-\bar{M} \bar{\theta}^{2}}{g^{2}}
$$

Since the gauge field strength tensors $W_{\alpha}\left(\bar{W}_{\alpha}\right)$ are chiral (antichiral) superfields, they enter into the chiral (antichiral) integrands in eq.(11), respectively. Correspondingly, the $M \theta^{2}$ term of eq. (12) contributes to the chiral integral, while the $\bar{M} \bar{\theta}^{2}$ term contributes to the antichiral one. There is no $\theta^{2} \bar{\theta}^{2}$ term in eq.(12), since it is neither chiral, no antichiral and gives no contribution to eq.(11). 
We depart here from the holomorphicity arguments [20]. Alternatively one should consider holomorphic and antiholomorphic gauge couplings and a separate non-chiral superfield to take care of the mixed term [7, 8]. This is where different approaches diverge. It does not lead, however, to any practical difference in applications within the PT.

Modifying the gauge coupling in the gauge part of the Lagrangian, one has to do the same in the gauge-fixing (5) and ghost (8) parts in order to preserve the BRST invariance. Here one has the integral over the whole superspace rather than the chiral one. This means that if one adds to eq.(12) a term proportional to $\theta^{2} \bar{\theta}^{2}$, it gives a nonzero contribution. Moreover, even if this term is not added, it reappears as a result of renormalization.

We suggest the following modification of eq.(12)

$$
\frac{1}{g^{2}} \rightarrow \frac{1}{\tilde{g}^{2}}=\frac{1-M \theta^{2}-\bar{M} \bar{\theta}^{2}-\Delta \theta^{2} \bar{\theta}^{2}}{g^{2}}
$$

which gives the final expression for the soft gauge coupling [4]

$$
\tilde{g}^{2}=g^{2}\left(1+M \theta^{2}+\bar{M} \bar{\theta}^{2}+2 M \bar{M} \theta^{2} \bar{\theta}^{2}+\Delta \theta^{2} \bar{\theta}^{2}\right) .
$$

It will be clear below that it is self-consistent to put $\Delta=0$ in the lowest order of perturbation theory, but it appears in higher orders due to renormalizations.

One has to take into account, however, that, since the gauge-fixing parameter $\xi$ may be considered as an additional coupling, it also becomes an external superfield and has to be modified. The soft expression can be written as

$$
\tilde{\xi}=\xi\left(1+x \theta^{2}+\bar{x} \bar{\theta}^{2}+(x \bar{x}+z) \theta^{2} \bar{\theta}^{2}\right),
$$

where parameters $x$ and $z$ can be obtained by solving the corresponding RG equation (see Appendix B).

Having this in mind, we perform the following modification of the gauge fixing condition (5) first used in [21]

$$
f \rightarrow \bar{D}^{2} \frac{V}{\sqrt{\tilde{\xi} \tilde{g}^{2}}}, \quad \bar{f} \rightarrow D^{2} \frac{V}{\sqrt{\tilde{\xi} \tilde{g}^{2}}} .
$$

Then, the gauge-fixing term (44) becomes

$$
\mathcal{L}_{\text {gauge-fixing }}=-\frac{1}{8} \int d^{2} \theta d^{2} \bar{\theta} \operatorname{Tr}\left(\bar{D}^{2} \frac{V}{\sqrt{\tilde{\xi} \tilde{g}^{2}}} D^{2} \frac{V}{\sqrt{\tilde{\xi} \tilde{g}^{2}}}\right) .
$$

This leads to the corresponding modification of the associated ghost term (6)

$$
\mathcal{L}_{\text {ghost }}=\int d^{2} \theta d^{2} \bar{\theta} \operatorname{Tr} \frac{1}{\sqrt{\tilde{\xi} \tilde{g}^{2}}}(b+\bar{b}) \mathcal{L}_{V / 2}\left[c+\bar{c}+\operatorname{coth}\left(\mathcal{L}_{V / 2}\right)(c-\bar{c})\right] .
$$

To understand the meaning of the $\Delta$ term, consider the quadratic part of the ghost Lagrangian (18)

$$
\mathcal{L}_{\text {ghost }}^{(2)}=\int d^{4} \theta \operatorname{Tr} \frac{1}{\sqrt{\xi g^{2}}}\left(1-\frac{1}{2} M \xi \theta^{2}-\frac{1}{2} \bar{M} \xi \bar{\theta}^{2}-\frac{1}{2} \Delta \xi \theta^{2} \bar{\theta}^{2}\right)(b+\bar{b})(c-\bar{c})
$$




$$
\begin{aligned}
& =\int d^{2} \theta d^{2} \bar{\theta} \operatorname{Tr} \frac{1}{\sqrt{\xi g^{2}}}\left(1-\frac{1}{2} \Delta \xi \theta^{2} \bar{\theta}^{2}\right)(b+\bar{b})(c-\bar{c}) \\
& -\frac{1}{2} \int d^{2} \theta \operatorname{Tr} \frac{1}{\sqrt{\xi g^{2}}} M \xi b c+\frac{1}{2} \int d^{2} \bar{\theta} \operatorname{Tr} \frac{1}{\sqrt{\xi g^{2}}} \bar{M} \xi \bar{b} \bar{c}
\end{aligned}
$$

where we have used the explicit form of $\tilde{\xi}$ given in Appendix B.

If one compares this expression with the usual Lagrangian for the matter fields (11), one finds an obvious identification of the second line with the soft scalar mass term and the third line with the mass term in a superpotential. Thus, $\Delta$ plays the role of a soft mass providing the splitting in the ghost supermultiplet.

The other place where the $\Delta$-term appears is the gauge-fixing term (17). Here it manifests itself as a soft mass of the auxiliary gauge field, one of the scalar components of the gauge superfield $V$.

To see this, consider the gauge-fixing term (17) in more detail. Expanding the vector superfield $V(x, \theta, \bar{\theta})$ in components

$$
\begin{aligned}
& V(x, \theta, \bar{\theta})=\mathbb{C}(x)+i \theta \chi(x)-i \bar{\theta} \bar{\chi}(x)+\frac{i}{2} \theta \theta N(x)-\frac{i}{2} \bar{\theta} \bar{\theta} \bar{N}(x)-\theta \sigma^{\mu} \bar{\theta} v_{\mu}(x) \\
& +i \theta \theta \bar{\theta}\left[\bar{\lambda}(x)+\frac{i}{2} \bar{\sigma}^{\mu} \partial_{\mu} \chi(x)\right]-i \bar{\theta} \bar{\theta} \theta\left[\lambda+\frac{i}{2} \sigma^{\mu} \partial_{\mu} \bar{\chi}(x)\right]+\frac{\theta \theta \bar{\theta} \bar{\theta}}{2}\left[D(x)-\frac{1}{2} \square \mathbb{C}(x)\right] .
\end{aligned}
$$

and substituting it into eq.(17) one finds

$$
\begin{aligned}
& \mathcal{L}_{\text {gauge-fixing }}=\frac{1}{2 \xi g^{2}}\left[-\left(D-\square \mathbb{C}-\Delta \xi \mathbb{C}+\frac{i}{2} M \xi \bar{N}-\frac{i}{2} \bar{M} \xi N\right)^{2}-\left(\partial^{\mu} v_{\mu}\right)^{2}\right. \\
& +(\bar{N}-i \bar{M} \xi \mathbb{C}) \square(N+i M \xi \mathbb{C})-i\left(\lambda+\frac{1}{2} \bar{M} \xi \chi\right) \sigma^{\mu} \partial_{\mu}\left(\bar{\lambda}+\frac{1}{2} M \xi \bar{\chi}\right) \\
& \left.-\left(\lambda+\frac{1}{2} \bar{M} \xi \chi\right) \square \chi-\left(\bar{\lambda}+\frac{1}{2} M \xi \bar{\chi}\right) \square \bar{\chi}-i \square \chi \sigma^{\mu} \partial_{\mu} \bar{\chi}\right] .
\end{aligned}
$$

One can see from eq.(20) that the parameter $M$, besides being the gaugino soft mass, plays the role of a mass of the auxiliary field $\chi$, while $\Delta$ is the soft mass of the auxiliary fields $N$ and $\mathbb{C}$. All these fields are unphysical degrees of freedom of the gauge superfield. They are absent in the Wess-Zumino gauge, however, when the gauge-fixing condition is chosen in supersymmetric form (4), this gauge is no longer possible, and the auxiliary fields $\chi, N$, and $\mathbb{C}$ survive. Thus, the extra $\Delta$ term is associated with unphysical, ghost, degrees of freedom, just like in the component approach, one has the mass of unphysical $\epsilon$-scalars 22. When we go down with energy, all massive fields decouple, and we get the usual nonsupersymmetric Yang-Mills theory.

The $\Delta$-term is renormalized and obeys its own RG equation which can be obtained from the corresponding expression for the gauge coupling via Grassmannian expansion. In due course of renormalization, this term is mixing with the soft masses of scalar superpartners and gives an additional term in RG equations for the latter.

In component formalism one has a similar term. In [10, 23], the dimensional reduction (DRED) regularization is used. In this case, one is bounded to introduce the so-called

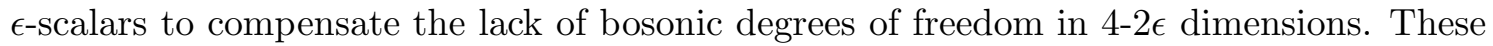
$\epsilon$-scalars in due course of renormalization acquire a soft mass that enters into the RG 
equations for soft masses of physical scalar particles. This problem has been discussed in [24. If one gets rid of the $\epsilon$-scalar mass by changing the renormalization scheme, DRED $\rightarrow$ DRED $^{\prime}$, there appears an additional term in RG equations for the soft scalar masses [25, 26] called X [10] which usually coincides with our $\Delta$.

Besides the modification of the gauge coupling the soft terms (10) imply the modification of the Yukawa ones and the mass term

$$
y^{i j k} \rightarrow \tilde{y}^{i j k}=y^{i j k}-A^{i j k} \eta, \quad M^{i j} \rightarrow \tilde{M}^{i j}=M^{i j}-B^{i j} \eta, \quad+\text { h.c. }
$$

There is, however, also the soft mass term $\left(m^{2}\right)_{i}^{k}$. To take care of it we absorb the multiplier $\left(\delta_{i}^{k}-\left(m^{2}\right)_{i}^{k} \eta \bar{\eta}\right)$ into the redefinition of the fields $\Phi$ and $\bar{\Phi}$. This results in the additional modification of the Yukawa couplings and the mass term

$$
\begin{aligned}
& y^{i j k} \rightarrow \tilde{y}^{i j k}=y^{i j k}-A^{i j k} \eta+\frac{1}{2}\left(y^{n j k}\left(m^{2}\right)_{n}^{i}+y^{i n k}\left(m^{2}\right)_{n}^{j}+y^{i j n}\left(m^{2}\right)_{n}^{k}\right) \eta \bar{\eta}, \\
& M^{i j} \rightarrow \tilde{M}^{i j}=M^{i j}-B^{i j} \eta+\frac{1}{2}\left(M^{n k}\left(m^{2}\right)_{n}^{i}+M^{i n}\left(m^{2}\right)_{n}^{k}\right) \eta \bar{\eta} .
\end{aligned}
$$

This completes our set of substitutions.

At the end of this section, we would like to comment on the BRST invariance in a softly broken SUSY theory. The BRST transformations (9) due to our choice of normalization of the gauge and ghost fields do not depend on the gauge coupling. Hence, in a softly broken theory they remain unchanged. One can easily check that, despite the substitution $g^{2} \rightarrow \tilde{g}^{2}$ and $\xi \rightarrow \tilde{\xi}$, the softly broken SUSY theory remains BRST invariant 21].

\section{Renormalizations in a Softly Broken SUSY Theory}

The modifications of the couplings introduced above are valid not only for the classical Lagrangian but also for the quantum one. As follows from the analysis of the Feynman diagrams in superspace 27] the modification of the Feynman rules due to the soft terms does not influence the UV divergent part of the effective action [1]. This justifies the statement made above concerning the UV singular part of the effective action. The following theorem is valid:

The theorem Let a rigid theory be renormalized via introduction of the renormalization constants $Z_{i}$, defined within some minimal subtraction massless scheme. Then, a softly broken theory is renormalized via introduction of the renormalization superfields $\tilde{Z}_{i}$ which are related to $Z_{i}$ by the coupling constant redefinition

$$
\tilde{Z}_{i}\left(g^{2}, y, \bar{y}\right)=Z_{i}\left(\tilde{g}^{2}, \tilde{y}, \tilde{\bar{y}}\right)
$$

where the redefined couplings are

$$
\begin{aligned}
\tilde{g}_{i}^{2} & =g_{i}^{2}\left(1+M_{i} \eta+\bar{M}_{i} \bar{\eta}+\left(2 M_{i} \bar{M}_{i}+\Delta_{i}\right) \eta \bar{\eta}\right), \\
\tilde{y}^{i j k} & =y^{i j k}-A^{i j k} \eta+\frac{1}{2}\left(y^{n j k}\left(m^{2}\right)_{n}^{i}+y^{i n k}\left(m^{2}\right)_{n}^{j}+y^{i j n}\left(m^{2}\right)_{n}^{k}\right) \eta \bar{\eta}, \\
\tilde{\bar{y}}_{i j k} & =\bar{y}_{i j k}-\bar{A}_{i j k} \bar{\eta}+\frac{1}{2}\left(y_{n j k}\left(m^{2}\right)_{i}^{n}+y_{i n k}\left(m^{2}\right)_{j}^{n}+y_{i j n}\left(m^{2}\right)_{k}^{n}\right) \eta \bar{\eta},
\end{aligned}
$$

Eqs.(22-24) lead to a finite renormalized softly broken SUSY theory. However, in practice it is more convenient to consider not the renormalization constants $Z_{i}$ but the 
RG equations directly. Differentiating the renormalization constants $\tilde{Z}_{i}$ with respect to a scale one gets the RG functions for the soft terms of a broken theory in terms of unbroken one. The resulting soft term $\beta$ functions are summarized below.

\section{Summary of the Soft Term Renormalizations}

\begin{tabular}{|c|c|}
\hline The Rigid Terms & The Soft Terms \\
\hline $\begin{array}{l}\beta_{\alpha_{i}}=\alpha_{i} \gamma_{\alpha_{i}} \\
\beta_{M}^{i j}=\frac{1}{2}\left(M^{i l} \gamma_{l}^{j}+M^{l j} \gamma_{l}^{i}\right) \\
\beta_{y}^{i j k}=\frac{1}{2}\left(y^{i j l} \gamma_{l}^{k}+\text { perm's }\right) \\
\quad \Uparrow \\
\text { chiral anomalous dim. }\end{array}$ & $\begin{array}{l}\beta_{M_{A i}}=D_{1} \gamma_{\alpha i} \\
\beta_{B}^{i j}=\frac{1}{2}\left(B^{i l} \gamma_{l}^{j}+B^{l j} \gamma_{l}^{i}\right)-\left(M^{i l} D_{1} \gamma_{l}^{j}+M^{l j} D_{1} \gamma_{l}^{i}\right) \\
\beta_{A}^{i j k}=\frac{1}{2}\left(A^{i j l} \gamma_{l}^{k}+\text { perm's }\right)-\left(y^{i j l} D_{1} \gamma_{l}^{k}+\text { perm's }\right) \\
\left(\beta_{m^{2}}\right)_{j}^{i}=D_{2} \gamma_{j}^{i} \\
\beta_{\Sigma_{\alpha_{i}}}=D_{2} \gamma_{\alpha_{i}}\end{array}$ \\
\hline $\begin{aligned} D_{1} & =M_{A_{i}} \alpha_{i} \frac{\partial}{\partial \alpha_{i}}-A^{i j k} \bar{\partial} \\
D_{2}= & \bar{D}_{1} D_{1}+\Sigma_{\alpha_{i}} \alpha_{i} \frac{\partial}{\partial \alpha_{i}} \\
& \quad+y_{a b c} \frac{\partial}{\partial y_{n b c}}+? \\
\Sigma_{\alpha_{i}} & =M_{A_{i}} \bar{M}_{A_{i}}+\Delta_{i}\end{aligned}$ & $\begin{array}{l}\frac{\bar{D}_{1}}{j k}, M_{A_{i}} \alpha_{i} \frac{\partial}{\partial \alpha_{i}}-A_{i j k} \frac{\partial}{\partial y_{i j k}} \\
\frac{1}{2}\left(m^{2}\right)_{n}^{a}\left(y^{n b c} \frac{\partial}{\partial y^{a b c}}+y^{b n c} \frac{\partial}{\partial y^{b a c}}+y^{b c n} \frac{\partial}{\partial y^{b c a}}\right. \\
\left.\frac{\partial}{\partial y_{b n c}}+y_{b c a} \frac{\partial}{\partial y_{b c n}}\right)\end{array}$ \\
\hline
\end{tabular}

Later on we consider some examples of the application of these formulas.

\section{Grassmannian Taylor Expansion}

We demonstrate now how the RG equations for the soft terms appear via Grassmannian Taylor expansion from those for the rigid couplings.

In what follows we would like to simplify the notations and consider numerical rather than tensorial couplings. When group structure and field content of the model are fixed, one has a set of gauge $\left\{g_{i}\right\}$ and Yukawa $\left\{y_{k}\right\}$ couplings. It is useful to consider the following rigid parameters $\alpha_{i} \equiv g_{i}^{2} / 16 \pi^{2}, Y_{k} \equiv y_{k}^{2} / 16 \pi^{2}$. Then eqs.(23.24) look like

$$
\begin{aligned}
\tilde{\alpha}_{i} & =\alpha_{i}\left(1+M_{i} \eta+\bar{M}_{i} \bar{\eta}+\left(M_{i} \bar{M}_{i}+\Sigma_{i}\right) \eta \bar{\eta}\right), \\
\tilde{Y}_{k} & =Y_{k}\left(1+A_{k} \eta+\bar{A}_{k} \bar{\eta}+\left(A_{k} \bar{A}_{k}+\Sigma_{k}\right) \eta \bar{\eta}\right),
\end{aligned}
$$

where to standardize the notations we have redefined parameter A: $A \rightarrow A y$ in a usual way and have changed the sign of A to match it with the gauge soft terms. Here $\Sigma_{k}$ stands for a sum of $m^{2}$ soft terms, one for each leg in the Yukawa vertex and $\Sigma_{i}=M_{i} \bar{M}_{i}+\Delta_{i}$.

Now the RG equation for a rigid theory can be written in a universal form

$$
\dot{a}_{i}=a_{i} \gamma_{i}(a), \quad a_{i}=\left\{\alpha_{i}, Y_{k}\right\}
$$

where $\gamma_{i}(a)$ stands for a sum of corresponding anomalous dimensions. In the same notation the soft terms (25) take the form

$$
\tilde{a}_{i}=a_{i}\left(1+m_{i} \eta+\bar{m}_{i} \bar{\eta}+S_{i} \eta \bar{\eta}\right),
$$


where $m_{i}=\left\{M_{i}, A_{k}\right\}$ and $S_{i}=\left\{M_{i} \bar{M}_{i}+\Sigma_{i}, A_{k} \bar{A}_{k}+\Sigma_{k}\right\}$.

Substituting eq.(27) into eq.(26) and expanding over $\eta$ and $\bar{\eta}$ one can get the RG equations for the soft terms

$$
\dot{\tilde{a}}_{i}=\tilde{a}_{i} \gamma_{i}(\tilde{a}),
$$

Consider first the F-terms. Expanding over $\eta$ one has

$$
\dot{m}_{i}=\left.\gamma_{i}(\tilde{a})\right|_{F}=\sum_{j} a_{j} \frac{\partial \gamma_{i}}{\partial a_{j}} m_{j} \equiv D_{1} \gamma_{i}
$$

This is just the RG equation for the soft terms $M_{i}$ and $A_{k}$ [6, 1]. Proceeding the same way for the D-terms and substituting $S_{i}=m_{i} \bar{m}_{i}+\Sigma_{i}$ one has the RG equation for the mass terms

$$
\dot{\Sigma}_{i}=\left.\gamma_{i}(\tilde{a})\right|_{D}=\sum_{j} a_{j} \frac{\partial \gamma_{i}}{\partial a_{j}}\left(m_{j} m_{j}+\Sigma_{j}\right)+\sum_{j, k} a_{j} a_{k} \frac{\partial^{2} \gamma_{i}}{\partial a_{j} \partial a_{k}} m_{j} m_{k} \equiv D_{2} \gamma_{i} .
$$

One can also obtain the RG equation for the individual soft masses out of field renormalization. Consider for this purpose the chiral Green function in a rigid theory. It obeys the following RG relation

$$
<\Phi_{i} \bar{\Phi}_{i}>=<\Phi_{i} \bar{\Phi}_{i}>_{0} e^{\int_{0}^{t} \gamma_{i}\left(a\left(t^{\prime}\right)\right) d t^{\prime}} .
$$

Making the substitution

$$
<\Phi_{i} \bar{\Phi}_{i}>\rightarrow<\Phi_{i} \bar{\Phi}_{i}>\left(1+m_{i}^{2} \eta \bar{\eta}\right), \quad a \rightarrow \tilde{a},
$$

and expanding over $\eta \bar{\eta}$ ( since it stands under the full Grassmann integral only D-term contributes) one has

$$
m_{i}^{2}=m_{i 0}^{2}+\left.\int_{0}^{t} d t^{\prime} \gamma_{i}\left(\tilde{a}\left(t^{\prime}\right)\right)\right|_{D} .
$$

Differentiating this relation with respect to $t$ leads to the RG equation for the soft mass

$$
\dot{m}^{2}{ }_{i}=D_{2} \gamma_{i}(a)
$$

\section{Illustration}

Consider, as an illustration of the above formulas, the simplest case of a pure gauge theory [3]. In a rigid theory the coupling is renormalized as

$$
\alpha^{\text {Bare }}=Z_{\alpha} \alpha, \quad \alpha \equiv g^{2} / 16 \pi^{2} .
$$

Making the substitution $\alpha \rightarrow \tilde{\alpha}$ one gets $\tilde{\alpha}^{\text {Bare }}=\tilde{Z}_{\alpha} \tilde{\alpha}$ or (up to linear terms in $\eta$ )

$$
\alpha^{\text {Bare }}\left(1+M_{A}^{\text {Bare }} \eta\right)=\alpha\left(1+M_{A} \eta\right) Z_{\alpha}\left(\alpha\left(1+M_{A} \eta\right)\right) .
$$


After expansion over $\eta$ this leads to equations

$$
\begin{aligned}
\alpha^{\text {Bare }} & =\alpha Z_{\alpha}(\alpha), \\
M_{A}^{\text {Bare }} \alpha^{\text {Bare }} & =M_{A} \alpha Z_{\alpha}(\alpha)+\alpha D_{1} Z_{\alpha},
\end{aligned}
$$

where $D_{1}=M_{A} \alpha \frac{\partial}{\partial \alpha}$ is the differential operator extracting linear terms over $\eta$. As a result, we get the bare mass

$$
M_{A}^{\text {Bare }}=M_{A}+D_{1} \ln Z_{\alpha} .
$$

Differentiating eq.(36) with respect to a scale, one gets

$$
\beta_{\alpha}=\alpha \gamma_{\alpha}, \quad \beta_{M_{A}}=D_{1} \gamma_{\alpha}
$$

where $\gamma_{\alpha}$ is the gauge field anomalous dimension $\gamma_{\alpha}=-d \log Z_{\alpha} / d \log \mu^{2}$.

In fact, one does not need eq.(37) to get the $\mathrm{RG}$ equation form the gaugino mass and can get the same formulas (37) starting directly from the RG equation for $\alpha$ as shown above. One can go even further and consider a solution to the RG equation in a rigid theory.

Indeed, let us take a solution to the RG equation for the coupling written in quadratures

$$
\int_{\alpha_{0}}^{\alpha} \frac{d \alpha^{\prime}}{\beta\left(\alpha^{\prime}\right)}=\ln \frac{Q^{2}}{\mu^{2}}
$$

Performing the replacement of the coupling one gets

$$
\int_{\alpha_{0}\left(1+M_{A 0} \eta+\ldots\right)}^{\alpha\left(1+M_{A} \eta+\ldots\right)} \frac{d \alpha^{\prime}}{\beta\left(\alpha^{\prime}\right)}=\ln \frac{Q^{2}}{\mu^{2}}
$$

which after expansion over $\eta$ leads to the solution for the soft mass term

$$
\frac{\alpha M_{A}}{\beta(\alpha)}=\frac{\alpha M_{A 0}}{\beta\left(\alpha_{0}\right)} \Rightarrow M_{A}=c_{1} \frac{\beta(\alpha)}{\alpha}=c_{1} \gamma(\alpha),
$$

where $\alpha$ is taken from eq. (38). Thus, the solution for the gaugino mass term directly follows from the one for the rigid coupling. Eq.(40) is the first example of RG invariants first found in 28] on different grounds. Following our approach one can construct the other ones 29]. For example, one can continue the expansion up to D-terms in eq.(39), which gives a solution for the $\Delta$ term

$$
\Delta=c_{2} \gamma(\alpha)-c_{1} \alpha \gamma^{\prime}(\alpha) \gamma(\alpha)
$$

\section{Examples}

\subsection{General gauge theory}

In the one-loop order the rigid $\beta$ functions are (for simplicity, we consider the case of a single gauge coupling)

$$
\begin{aligned}
\beta_{\alpha} & =\alpha \gamma_{\alpha}, \quad \gamma_{\alpha}^{(1)}=\alpha Q, \quad Q=T(R)-3 C(G) \\
\beta_{y}^{i j k} & =\frac{1}{2}\left(y^{i j l} \gamma_{l}^{k}+p^{2} m^{\prime} s\right), \quad \gamma_{j}^{i(1)}=\frac{1}{2} y^{i k l} y_{j k l}-2 \alpha C(R)_{j}^{i},
\end{aligned}
$$


where $T(R), C(G)$ and $C(R)$ are the Casimir operators of the gauge group defined by

$$
T(R) \delta_{A B}=\operatorname{Tr}\left(R_{A} R_{B}\right), \quad C(G) \delta_{A B}=f_{A C D} f_{B C D}, \quad C(R)_{j}^{i}=\left(R_{A} R_{A}\right)_{j}^{i} .
$$

Applying our algorithm, this leads to the following soft $\beta$ functions:

$$
\begin{aligned}
\beta_{M_{A}}^{(1)} & =\alpha M_{A} Q \\
\beta_{B}^{i j(1)} & =\frac{1}{2} B^{i l}\left(\frac{1}{2} y^{j k m} y_{l k m}-2 \alpha C(R)_{l}^{j}\right) \\
& +M^{i l}\left(\frac{1}{2} A^{j k m} y_{l k m}+2 \alpha M_{A} C(R)_{l}^{j}\right)+(i \leftrightarrow j), \\
\beta_{A}^{i j k(1)} & =\frac{1}{2} A^{i j l}\left(\frac{1}{2} y^{k m n} y_{l m n}-2 \alpha C(R)_{l}^{k}\right) \\
& +y^{i j l}\left(\frac{1}{2} A^{k m n} y_{l m n}+2 \alpha M_{A} C(R)_{l}^{k}\right)+(i \leftrightarrow j, k), \\
{\left[\beta_{m^{2}}\right]_{j}^{i(1)} } & =\frac{1}{2} A^{i k l} A_{j k l}-4 \alpha M_{A}^{2} C(R)_{j}^{i} \\
& +\frac{1}{4} y^{n k l}\left(m^{2}\right)_{n}^{i} y_{j k l}+\frac{1}{4} y^{i k l}\left(m^{2}\right)_{j}^{n} y_{n k l}+\frac{4}{4} y^{i s l}\left(m^{2}\right)_{s}^{k} y_{j k l} .
\end{aligned}
$$

We used here the fact that in the given order the solution for $\Sigma_{\alpha}$ is $\Sigma_{\alpha}=M_{A} \bar{M}_{A}$.

In two loops the rigid anomalous dimensions are

$$
\begin{aligned}
\gamma_{\alpha}^{(2)} & =2 \alpha^{2} C(G) Q-\frac{2 \alpha}{r} C(R)_{j}^{i}\left(\frac{1}{2} y^{j k l} y_{i k l}-2 \alpha C(R)_{i}^{j}\right), \quad r=\operatorname{dim} G=\delta_{A A}, \\
\gamma_{j}^{i(2)} & =-\left(y^{i m p} y_{j m n}+2 \alpha C(R)_{j}^{p} \delta_{n}^{i}\right)\left(\frac{1}{2} y^{n k l} y_{p k l}-2 \alpha C(R)_{p}^{n}\right)+2 \alpha^{2} Q C(R)_{j}^{i} .
\end{aligned}
$$

In this case, again the solution for the ghost mass $\Delta_{\alpha}$ can be found analytically [俩] and coincides with the mass of $\epsilon$-scalars [30]

$$
\Sigma_{\alpha}^{(2)}=\Delta_{\alpha}^{(2)}=-2 \alpha\left[\frac{1}{r}\left(m^{2}\right)_{j}^{i} C(R)_{i}^{j}-M_{A}^{2} C(G)\right] .
$$

Then, the soft renormalizations are as follows:

$$
\begin{aligned}
\beta_{M_{A}}^{(2)}= & 4 \alpha^{2} M_{A} C(G) Q-\frac{2 \alpha M_{A}}{r} C(R)_{j}^{i}\left(\frac{1}{2} y^{j k l} y_{i k l}-2 \alpha C(R)_{i}^{j}\right) \\
& +\frac{2 \alpha}{r} C(R)_{j}^{i}\left(\frac{1}{2} A^{j k l} y_{i k l}+2 \alpha M_{A} C(R)_{i}^{j}\right), \\
\beta_{B}^{i j(2)}= & -\frac{1}{2} B^{i l}\left(y^{j k p} y_{l k n}+2 \alpha C(R)_{l}^{p} \delta_{n}^{j}\right)\left(\frac{1}{2} y^{n s t} y_{p s t}-2 \alpha C(R)_{p}^{n}\right) \\
& -M^{i l}\left(A^{j k p} y_{l k n}-2 \alpha M_{A} C(R)_{l}^{p} \delta_{n}^{j}\right)\left(\frac{1}{2} y^{n s t} y_{p s t}-2 \alpha C(R)_{p}^{n}\right) \\
& -M^{i l}\left(y^{j k p} y_{l k n}+2 \alpha C(R)_{l}^{p} \delta_{n}^{j}\right)\left(\frac{1}{2} A^{n s t} y_{p s t}+2 \alpha M_{A} C(R)_{p}^{n}\right) \\
& +B^{i l} \alpha^{2} Q C(R)_{l}^{j}-4 M^{i l} \alpha^{2} Q C(R)_{l}^{j} M_{A}+(i \leftrightarrow j), \\
\beta_{A}^{i j k(2)}= & -\frac{1}{2} A^{i j l}\left(y^{k m p} y_{l m n}+2 \alpha C(R)_{l}^{p} \delta_{n}^{k}\right)\left(\frac{1}{2} y^{n s t} y_{p s t}-2 \alpha C(R)_{p}^{n}\right) \\
& +A^{i j l} \alpha^{2} Q C(R)_{l}^{k}-4 y^{i j l} \alpha^{2} Q C(R)_{l}^{j} M_{A}
\end{aligned}
$$




$$
\begin{aligned}
& -y^{i j l}\left(A^{k m p} y_{l m n}-2 \alpha M_{A} C(R)_{l}^{p} \delta_{n}^{k}\right)\left(\frac{1}{2} y^{n s t} y_{p s t}-2 \alpha C(R)_{p}^{n}\right) \\
& -y^{i j l}\left(y^{k m p} y_{l m n}+2 \alpha C(R)_{l}^{p} \delta_{n}^{k}\right)\left(\frac{1}{2} A^{n s t} y_{p s t}+2 \alpha M_{A} C(R)_{p}^{n}\right) \\
& +(i \leftrightarrow j)+(i \leftrightarrow k) \\
{\left[\beta_{m^{2}}\right]_{j}^{i(2)}=} & -\left(A^{i k p} A_{j k n}+\frac{1}{2}\left(m^{2}\right)_{l}^{i} y^{l k p} y_{j k n}+\frac{1}{2} y^{i k p} y_{l k n}\left(m^{2}\right)_{j}^{l}\right. \\
& +\frac{2}{2} y^{i l p}\left(m^{2}\right)_{l}^{s} y_{j s n}+\frac{1}{2} y^{i k s}\left(m^{2}\right)_{s}^{p} y_{j k n}+\frac{1}{2} y_{i k p}\left(m^{2}\right)_{n}^{s} y_{j k s} \\
& \left.+4 \alpha M_{A}^{2} C(R)_{j}^{p} \delta_{n}^{i}\right)\left(\frac{1}{2} y^{n s t} y_{p s t}-2 \alpha C(R)_{p}^{n}\right) \\
& -\left(y^{i k p} y_{j k n}+2 \alpha C(R)_{j}^{p} \delta_{n}^{i}\right)\left(\frac{1}{2} A^{n s t} A_{p s t}+\frac{1}{4}\left(m^{2}\right)_{l}^{k} y^{l s t} y_{p s t}\right. \\
& \left.+\frac{1}{4} y^{n s t} y_{l s t}\left(m^{2}\right)_{p}^{l}+\frac{4}{4} y^{n l t}\left(m^{2}\right)_{l}^{s} y_{p s t}-2 \alpha M_{A}^{2} C(R)_{p}^{n}\right) \\
& -\left(A^{i k p} y_{j k n}-2 \alpha M_{A} C(R)_{j}^{p} \delta_{n}^{i}\right)\left(\frac{1}{2} y^{n s t} A_{p s t}+2 \alpha M_{A} C(R)_{p}^{n}\right) \\
& -\left(y^{i k p} A_{j k n}-2 \alpha M_{A} C(R)_{j}^{p} \delta_{n}^{i}\right)\left(\frac{1}{2} A^{n s t} y_{p s t}+2 \alpha M_{A} C(R)_{p}^{n}\right) \\
& +12 \alpha^{2} M_{A}^{2} Q C(R)_{j}^{i}+4 \alpha^{2} C(R)_{j}^{i}\left[\frac{1}{r}\left(m^{2}\right)_{l}^{k} C(R)_{k}^{l}-M^{2} C(G)\right],
\end{aligned}
$$

where the last term is an extra contribution due to nonzero $\Delta_{i}^{(2)}$ in (47).

To demonstrate the power of the proposed algorithm, we calculate the three loop gaugino mass renormalization out of a gauge $\beta$ function. One has in three loops 15.

$$
\begin{aligned}
\gamma_{\alpha}^{(3)} & =\alpha^{3} C(G) Q[4 C(G)-Q]-\frac{6}{r} \alpha^{3} Q C(R)_{j}^{i} C(R)_{i}^{j} \\
& +\frac{3}{r} \alpha^{2}\left(y^{i k l} y_{j k l}-4 \alpha C(R)_{j}^{i}\right) C(R)_{s}^{j} C(R)_{i}^{s}-\frac{2}{r} \alpha^{2} C(G)\left(y^{i k l} y_{j k l}\right. \\
& \left.-4 \alpha C(R)_{j}^{i}\right) C(R)_{i}^{j}+\frac{3}{2 r} \alpha y^{i k m} y_{j k n}\left(y^{n s t} y_{m s t}-4 \alpha C(R)_{m}^{n}\right) C(R)_{i}^{j} \\
& +\frac{1}{4 r} \alpha\left(y^{i k l} y_{j k l}-4 \alpha C(R)_{j}^{i}\right)\left(y^{j s t} y_{p s t}-4 \alpha C(R)_{p}^{j}\right) C(R)_{i}^{p} .
\end{aligned}
$$

The corresponding gaugino mass renormalization is

$$
\begin{aligned}
\beta_{M_{A}}^{(3)} & =3 \alpha^{3} C(G) Q[4 C(G)-Q] M_{A}-\frac{18}{r} \alpha^{3} Q C(R)_{j}^{i} C(R)_{i}^{j} M_{A} \\
& +\frac{6}{r} \alpha^{2}\left(y^{i k l} y_{j k l}-4 \alpha C(R)_{j}^{i}\right) C(R)_{s}^{j} C(R)_{i}^{s} M_{A}-\frac{3}{r} \alpha^{2}\left(A^{i k l} y_{j k l}\right. \\
& \left.+4 \alpha C(R)_{j}^{i} M_{A}\right) C(R)_{s}^{j} C(R)_{i}^{s}-\frac{4}{r} \alpha^{2} C(G)\left(y^{i k l} y_{j k l}-4 \alpha C(R)_{j}^{i}\right) C(R)_{i}^{j} M_{A} \\
& +\frac{2}{r} \alpha^{2} C(G)\left(A^{i k l} y_{j k l}+4 \alpha C(R)_{j}^{i} M_{A}\right) C(R)_{i}^{j}+\frac{3}{2 r} \alpha y^{i k m} y_{j k n}\left(y^{n s t} y_{m s t}\right. \\
& \left.-4 \alpha C(R)_{m}^{n}\right) C(R)_{i}^{j} M_{A}-\frac{3}{2 r} \alpha A^{i k m} y_{j k n}\left(y^{n s t} y_{m s t}-4 \alpha C(R)_{m}^{n}\right) C(R)_{i}^{j} \\
& -\frac{3}{2 r} \alpha y^{i k m} y_{j k n}\left(A^{n s t} y_{m s t}+4 \alpha C(R)_{m}^{n} M_{A}\right) C(R)_{i}^{j} \\
& +\frac{1}{4 r} \alpha\left(y^{i k l} y_{j k l}-4 \alpha C(R)_{j}^{i}\right)\left(y^{j s t} y_{p s t}-4 \alpha C(R)_{p}^{j}\right) C(R)_{i}^{p} M_{A}
\end{aligned}
$$




$$
\begin{aligned}
& -\frac{1}{4 r} \alpha\left(A^{i k l} y_{j k l}+4 \alpha C(R)_{j}^{i} M_{A}\right)\left(y^{j s t} y_{p s t}-4 \alpha C(R)_{p}^{j}\right) C(R)_{i}^{p} \\
& -\frac{1}{4 r} \alpha\left(y^{i k l} y_{j k l}-4 \alpha C(R)_{j}^{i}\right)\left(A^{j s t} y_{p s t}+4 \alpha C(R)_{p}^{j} M_{A}\right) C(R)_{i}^{p} .
\end{aligned}
$$

To argue that a solution for $\Delta_{i}$ exists in all orders of PT, one can consider the so-called NSVZ-scheme [31] where the anomalous dimension $\gamma_{\alpha}$ is known to all orders of PT

$$
\gamma_{\alpha}^{N S V Z}=\alpha \frac{Q-2 r^{-1} \operatorname{Tr}[\gamma C(R)]}{1-2 C(G) \alpha} .
$$

Then the solution for $\Delta_{\alpha}$ is

$$
\Delta_{\alpha}^{N S V Z}=-2 \alpha \frac{r^{-1} \operatorname{Tr}\left[m^{2} C(R)\right]-M_{A}^{2} C(G)}{1-2 C(G) \alpha} .
$$

and coincides with the for $\epsilon$-scalar mass [30, 32].

\subsection{The MSSM in low $\tan \beta$ regime}

Consider the MSSM in low $\tan \beta$ regime. One has three gauge and one Yukawa coupling. The one-loop RG equations are 33]

$$
\begin{aligned}
& \dot{\alpha}_{i}=-b_{i} \alpha_{i}^{2}, \quad b_{i}=\left(\frac{33}{5}, 1,-3\right), \quad i=1,2,3, \\
& \dot{Y}_{t}=Y_{t}\left(\frac{16}{3} \alpha_{3}+3 \alpha_{2}+\frac{13}{15} \alpha_{1}-6 Y_{t}\right),
\end{aligned}
$$

with the initial conditions: $\alpha_{i}(0)=\alpha_{0}, Y_{t}(0)=Y_{0}$ and $t=\ln \left(M_{X}^{2} / Q^{2}\right)$. Their solutions are given by 33

$$
\alpha_{i}(t)=\frac{\alpha_{0}}{1+b_{i} \alpha_{0} t}, \quad Y_{t}(t)=\frac{Y_{0} E(t)}{1+6 Y_{0} F(t)}
$$

where

$$
E(t)=\prod_{i}\left(1+b_{i} \alpha_{0} t\right)^{c_{i} / b_{i}}, \quad c_{i}=\left(\frac{13}{15}, 3, \frac{16}{3}\right), \quad F(t)=\int_{0}^{t} E\left(t^{\prime}\right) d t^{\prime} .
$$

To get the solutions for the soft terms it is enough to perform the substitution $\alpha \rightarrow \tilde{\alpha}$

and $Y \rightarrow \tilde{Y}$ and expand over $\eta$ and $\bar{\eta}$. Expanding the gauge coupling in (58) up to $\eta$ one has (hereafter we assume $M_{i 0}=M_{0}$ )

$$
\alpha_{i} M_{i}=\frac{\alpha_{0} M_{0}}{1+b_{i} \alpha_{0} t}-\frac{\alpha_{0} b_{i} \alpha_{0} M_{0} t}{\left(1+b_{i} \alpha_{0} t\right)^{2}}=\frac{\alpha_{0}}{1+b_{i} \alpha_{0} t} \cdot \frac{M_{0}}{1+b_{i} \alpha_{0} t}
$$

or

$$
M_{i}(t)=\frac{M_{0}}{1+b_{i} \alpha_{0} t}
$$

Performing the same expansion for the Yukawa coupling and using the relations

$$
\left.\frac{d \tilde{E}}{d \eta}\right|_{\eta}=M_{0} t \frac{d E}{d t},\left.\quad \frac{d \tilde{F}}{d \eta}\right|_{\eta}=M_{0}(t E-F)
$$


one finds a well known expression [33]

$$
A_{t}(t)=\frac{A_{0}}{1+6 Y_{0} F}+M_{0}\left(\frac{t}{E} \frac{d E}{d t}-\frac{6 Y_{0}}{1+6 Y_{0} F}(t E-F)\right) .
$$

To get the solution for the term $\Sigma_{t}=\tilde{m}_{t}^{2}+\tilde{m}_{Q}^{2}+m_{H_{2}}^{2}$ one has to make expansion over $\eta$ and $\bar{\eta}$. This can be done with the help of the following relations

$$
\left.\frac{d^{2} \tilde{E}}{d \eta d \bar{\eta}}\right|_{\eta, \bar{\eta}}=M_{0}^{2} \frac{d}{d t}\left(t^{2} \frac{d E}{d t}\right),\left.\quad \frac{d^{2} \tilde{F}}{d \eta d \bar{\eta}}\right|_{\eta, \bar{\eta}}=M_{0}^{2} t^{2} \frac{d E}{d t}
$$

and leads to [3]

$$
\begin{aligned}
\Sigma_{t}(t)= & \frac{\Sigma_{0}-A_{0}^{2}}{1+6 Y_{0} F}+\frac{\left(A_{0}-M_{0} 6 Y_{0}(t E-F)\right)^{2}}{\left(1+6 Y_{0} F\right)^{2}} \\
& +M_{0}^{2}\left[\frac{d}{d t}\left(\frac{t^{2}}{E} \frac{d E}{d t}\right)-\frac{6 Y_{0}}{1+6 Y_{0} F} t^{2} \frac{d E}{d t}\right] .
\end{aligned}
$$

With analytic solutions (60,61) one can analyze asymptotics and, in particular, find the so-called infrared quasi fixed points [34] which correspond to $Y_{0} \rightarrow \infty$

$$
\begin{aligned}
Y_{t}^{F P} & =\frac{E}{6 F}, \\
A_{t}^{F P} & =M_{0}\left(\frac{t}{E} \frac{d E}{d t}-\frac{t E-F}{F}\right), \\
\Sigma_{t}^{F P} & =M_{0}^{2}\left[\left(\frac{t E-F}{F}\right)^{2}+\frac{d}{d t}\left(\frac{t^{2}}{E} \frac{d E}{d t}\right)-\frac{t^{2}}{F} \frac{d E}{d t}\right] .
\end{aligned}
$$

However, the advantage of the Grassmannian expansion procedure is that one can perform it for fixed points as well. Thus the FP solutions (63, 64) can be directly obtained from a fixed point for the rigid Yukawa coupling (62) by Grassmannian expansion. This explains, in particular, why fixed point solutions for the soft couplings exist if they exist for the rigid ones and with the same stability properties [35].

\subsection{The MSSM in high $\tan \beta$ regime}

Consider the MSSM in high $\tan \beta$ regime. One has three gauge and three Yukawa couplings. The one-loop RG equations are 33]

$$
\dot{\alpha}_{i}=-b_{i} \alpha_{i}^{2}, \quad \dot{Y}_{k}=Y_{k}\left(\sum_{i} c_{k i} \alpha_{i}-\sum_{l} a_{k l} Y_{l}\right)
$$

where $i=1,2,3 ; k=t, b, \tau, \cdot \equiv d / d t, t=\log M_{G U T}^{2} / Q^{2}$ and

$$
\begin{aligned}
b_{i} & =\{33 / 5,1,-3\}, \quad a_{t l}=\{6,1,0\}, \quad a_{b l}=\{1,6,1\}, \quad a_{\tau l}=\{0,3,4\} \\
c_{t i} & =\{13 / 15,3,16 / 3\}, \quad c_{b i}=\{7 / 15,3,16 / 3\}, \quad c_{\tau i}=\{9 / 5,3,0\} .
\end{aligned}
$$


Despite a simple form of these equations, there is no explicit analytic solution similar to (58). One has either the iterative solution [36] or the approximate one [37]. In both the cases the Grassmannian expansion over $\eta$ leads to the corresponding solutions for the soft terms.

Consider first the iterative solution. It can be written as [36]

$$
\alpha_{i}=\frac{\alpha_{i}^{0}}{1+b_{i} \alpha_{i}^{0} t}, \quad Y_{k}=\frac{Y_{k}^{0} u_{k}}{1+a_{k k} Y_{k}^{0} \int_{0}^{t} u_{k}},
$$

where the functions $\left\{u_{k}\right\}$ obey the integral system of equations

$$
\begin{aligned}
u_{t} & =\frac{E_{t}}{\left(1+6 Y_{b}^{0} \int_{0}^{t} u_{b}\right)^{1 / 6}}, \quad u_{\tau}=\frac{E_{\tau}}{\left(1+6 Y_{b}^{0} \int_{0}^{t} u_{b}\right)^{1 / 2}} \\
u_{b} & =\frac{E_{b}}{\left(1+6 Y_{t}^{0} \int_{0}^{t} u_{t}\right)^{1 / 6}\left(1+4 Y_{\tau}^{0} \int_{0}^{t} u_{\tau}\right)^{1 / 4}}
\end{aligned}
$$

and the functions $E_{k}$ are given by $E_{k}=\prod_{i=1}^{3}\left(1+b_{i} \alpha_{i}^{0} t\right)^{c_{k i} / b_{i}}$.

Let us stress that eqs. (66) give the exact solution to eqs.(65), while the $u_{k}$ 's in eqs.(67), although solved formally in terms of the $E_{k}$ 's and $Y_{k}^{0}$ 's as continued integrated fractions, should in practice be solved iteratively.

To get the solutions for the soft terms it is enough to perform substitution $\alpha_{i} \rightarrow \tilde{\alpha}_{i}$ and $Y_{k} \rightarrow \tilde{Y}_{k}$ and expand over $\eta$ and $\bar{\eta}$. One has [38]:

$$
\begin{array}{r}
M_{i}=\frac{M_{i}^{0}}{1+b_{i} \alpha_{i}^{0} t}, \quad A_{k}=-e_{k}+\frac{A_{k}^{0} / Y_{k}^{0}+a_{k k} \int u_{k} e_{k}}{1 / Y_{k}^{0}+a_{k k} \int u_{k}}, \\
\Sigma_{k}=\xi_{k}+A_{k}^{2}+2 e_{k} A_{k}-\frac{\left(A_{k}^{0}\right)^{2} / Y_{k}^{0}-\Sigma_{k}^{0} / Y_{k}^{0}+a_{k k} \int u_{k} \xi_{k}}{1 / Y_{k}^{0}+a_{k k} \int u_{k}},
\end{array}
$$

where the new functions $e_{k}$ and $\xi_{k}$ have been introduced which obey the iteration equations. For illustration we present below the corresponding expressions for $e_{t}$ and $\xi_{t}$

$$
\begin{aligned}
e_{t}= & \frac{1}{E_{t}} \frac{d \tilde{E}_{t}}{d \eta}+\frac{A_{b}^{0} \int u_{b}-\int u_{b} e_{b}}{1 / Y_{b}^{0}+6 \int u_{b}} \\
\xi_{t}= & \frac{1}{E_{t}} \frac{d^{2} \tilde{E}_{t}}{d \eta d \bar{\eta}}+2 \frac{1}{E_{t}} \frac{d \tilde{E}_{t}}{d \eta} \frac{A_{b}^{0} \int u_{b}-\int u_{b} e_{b}}{1 / Y_{b}^{0}+6 \int u_{b}}+7 \frac{\left(A_{b}^{0} \int u_{b}-\int u_{b} e_{b}\right)^{2}}{\left(1 / Y_{b}^{0}+6 \int u_{b}\right)^{2}} \\
& -\frac{\left(\Sigma_{b}^{0}+\left(A_{b}^{0}\right)^{2}\right) \int u_{b}-2 A_{b}^{0} \int u_{b} e_{b}+\int u_{b} \xi_{b}}{1 / Y_{b}^{0}+6 \int u_{b}},
\end{aligned}
$$

where the variations of $\tilde{E}_{k}$ should be taken at $\eta=\bar{\eta}=0$ and are given by

$$
\begin{aligned}
& \left.\frac{1}{E_{k}} \frac{d \tilde{E}_{k}}{d \eta}\right|_{\eta, \bar{\eta}=0}=t \sum_{i=1}^{3} c_{k i} \alpha_{i} M_{i}^{0} \\
& \left.\frac{1}{E_{k}} \frac{d^{2} \tilde{E}_{k}}{d \eta d \bar{\eta}}\right|_{\eta, \bar{\eta}=0}=t^{2}\left(\sum_{i=1}^{3} c_{k i} \alpha_{i} M_{i}^{0}\right)^{2}+2 t \sum_{i=1}^{3} c_{k i} \alpha_{i}\left(M_{i}^{0}\right)^{2}-t^{2} \sum_{i=1}^{3} c_{k i} b_{i} \alpha_{i}^{2}\left(M_{i}^{0}\right)^{2} .
\end{aligned}
$$


When solving eqs.(67) and (69) in the $n$-th iteration one has to substitute in the r.h.s. the $(n-1)$-th iterative solution for all the corresponding functions.

The same procedure works for the approximate solutions. Once one gets an approximate solution for the Yukawa couplings, one immediately has those for the soft terms as well [37].

We consider as an illustration the approximate solution. It can be taken in the form [37]

$$
\begin{aligned}
Y_{t}^{a p p}(t)= & \frac{Y_{t 0} E_{t}(t)}{\left[1+\frac{7}{2}\left(Y_{t 0} F_{t}(t)+Y_{b 0} F_{b}(t)\right)\right]^{2 / 7}\left[1+7 Y_{t 0} F_{t}(t)\right]^{5 / 7}} \\
Y_{b}^{a p p}(t)= & \frac{Y_{b 0} E_{b}(t)}{\left[1+\frac{7}{2}\left(Y_{t 0} F_{t}(t)+Y_{b 0} F_{b}(t)\right)\right]^{2 / 7}\left[1+7 Y_{t 0} F_{t}(t)\right]^{2 / 7}}, \\
& \times \frac{1}{\left[1+\frac{7}{3}\left(3 Y_{b 0} F_{b}(t)+Y_{\tau 0} F_{\tau}\right)\right]^{3 / 7}}, \\
Y_{\tau}^{a p p}(t)= & \frac{Y_{\tau 0} E_{\tau}(t)}{\left[1+\frac{21}{4} Y_{\tau 0} F_{\tau}\right]^{4 / 7}\left[1+\frac{7}{3}\left(3 Y_{b 0} F_{b}(t)+Y_{\tau 0} F_{\tau}\right)\right]^{3 / 7}} .
\end{aligned}
$$

To demonstrate the accuracy of the approximate solution (70) and the efficiency of the Grassmannian expansion, we present in Fig.1 the comparison of numerical and approximate solutions for the Yukawa couplings of a rigid theory as well as the soft terms.

One can notice perfect agreement of numerical and analytical curves. Shown also are the fixed point behaviour, again for the Yukawa couplings and for the soft terms obtained via the expansion procedure for the approximate solutions (70). The numerical curves approach the analytically calculated FP's in the infrared region.

\subsection{Totally all loop finite $\mathrm{N}=1$ SUSY gauge theories}

Another example of application of the same procedure is the so-called finite field theories in the framework of SUSY GUTs. These are the theories where all the UV divergences cancel and hence all the $\beta$ functions vanish. This can be achieved in a rigid theory if the following two conditions are satisfied [39, 40]:

- The group representations are chosen in a way to obey the sum rule

$$
\sum T(R)=3 C_{2}(G)
$$

- The Yukawa couplings are the functions of the gauge one

$$
Y_{i}=Y_{i}(\alpha), Y_{i}(\alpha)=c_{1}^{i} \alpha+c_{2}^{i} \alpha^{2}+\ldots
$$

where the coefficients $c_{n}^{i}$ are calculated algebraically in the n-th order of PT.

To achieve the complete finiteness of the model including the soft terms, one has to modify the finiteness condition (72) as

$$
\tilde{Y}_{i}=Y_{i}(\tilde{\alpha})
$$

and perform the expansion over $\eta, \bar{\eta}$. This gives [2]

$$
\left\{\begin{array}{c}
A_{i}=-M_{A} \frac{d \ln Y_{i}}{d \ln \alpha}, \\
\Sigma_{i}=M_{A}^{2} \frac{d}{d \alpha} \alpha \frac{d \ln Y_{i}}{d \ln \alpha},
\end{array}\right.
$$



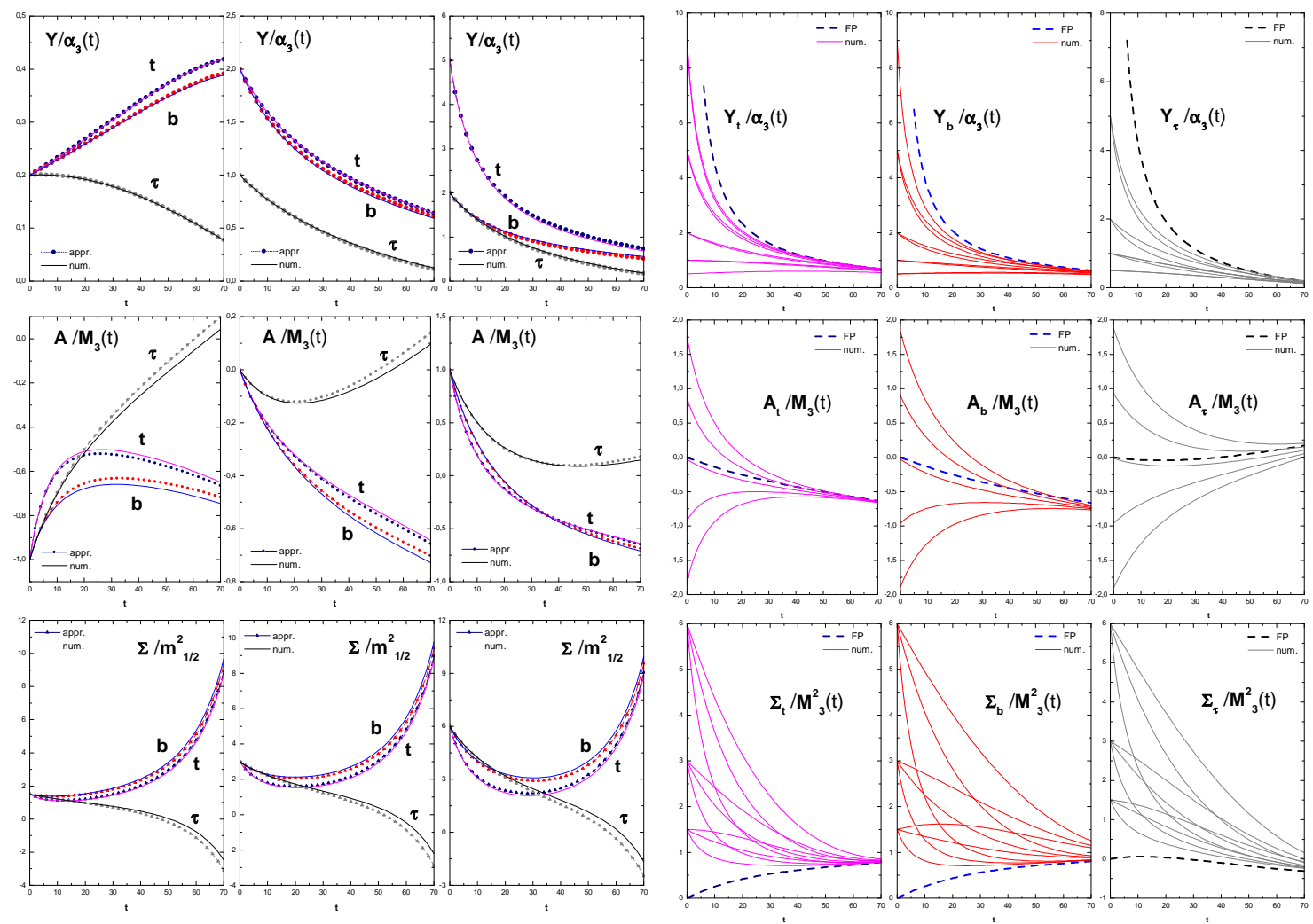

Figure 1: Comparison of numerical and approximate solutions. Dotted lines correspond to the analytical approximate solutions, solid lines to the numerical solution. Shown also are the infra-red quasi fixed points obtained via Grassmannian expansion and the numerical curves approaching them in the IR limit.

where $Y(\alpha)$ is assumed to be known from a rigid theory. These expressions lead to a totally finite softly broken SUSY field theory!

Alternatively one can formulate the same conditions in terms of the bare couplings. They are finite in this case. In dimensional regularization one has instead of eq.(72)

$$
Y_{i}^{\text {Bare }}=\alpha_{\text {Bare }} \cdot f_{i}(\varepsilon), \quad f_{i}(\varepsilon)=c_{i}^{(1)}+c_{i}^{(2)} \varepsilon+c_{i}^{(3)} \varepsilon^{2}+\ldots
$$

where the coefficients $c_{i}^{(n)}$ are in one-to-one correspondence to those in eq.(72). Replacing the couplings in eq.(75) in a usual way one finds that the function $f(\varepsilon)$ cancels and one has simple relations for the soft terms valid in all orders of PT [2]

$$
\tilde{Y}_{i}^{\text {Bare }}=\tilde{\alpha}_{\text {Bare }} \cdot f_{i}(\varepsilon) \Rightarrow\left\{\begin{array}{l}
A_{i}^{\text {Bare }}=-M_{A}^{\text {Bare }} \\
\Sigma_{i}^{\text {Bare }}=\left(M_{A}^{\text {Bare }}\right)^{2}
\end{array}\right.
$$

These relations for the bare quantities provide the vanishing of the $\beta$ functions for the soft terms. 


\subsection{N=2 SUSY Seiberg-Witten Theory}

Consider now the $\mathrm{N}=2$ supersymmetric gauge theory. The Lagrangian written in terms of $\mathrm{N}=2$ superfields is 41]:

$$
\mathcal{L}=\frac{1}{4 \pi} \mathcal{I} m \operatorname{Tr} \int d^{2} \theta_{1} d^{2} \theta_{2} \frac{1}{2} \tau \Psi^{2}
$$

where $\mathrm{N}=2$ chiral superfield $\Psi\left(y, \theta_{1}, \theta_{2}\right)$ is defined by constraints $\bar{D}_{\dot{\alpha}} \Psi=0$ and $\overline{\tilde{D}}_{\dot{\alpha}} \Psi=0$ and

$$
\tau=i \frac{4 \pi}{g^{2}}+\frac{\theta}{2 \pi}^{\text {topological }}
$$

The expansion of $\Psi$ in terms of $\theta_{2}$ can be written as

$$
\Psi\left(y, \theta_{1}, \theta_{2}\right)=\Psi^{(1)}\left(y, \theta_{1}\right)+\sqrt{2} \theta_{2}^{\alpha} \Psi_{\alpha}^{(2)}\left(y, \theta_{1}\right)+\theta_{2}^{\alpha} \theta_{2 \alpha} \Psi^{(3)}\left(y, \theta_{1}\right),
$$

where $y^{\mu}=x^{\mu}+i \theta_{1} \sigma^{\mu} \bar{\theta}_{1}+i \theta_{2} \sigma^{\mu} \bar{\theta}_{2}$ and $\Psi^{(k)}\left(y, \theta_{1}\right)$ are $\mathrm{N}=1$ chiral superfields.

The soft breaking of $\mathrm{N}=2 \mathrm{SUSY}$ down to $\mathrm{N}=0$ can be achieved by shifting the imaginary part of $\tau$ :

$$
\mathcal{I} m \tau \rightarrow \mathcal{I} m \tilde{\tau}=\mathcal{I} m \tau\left(1+M_{1} \theta_{1}^{2}+M_{2} \theta_{2}^{2}+M_{3} \theta_{1}^{2} \theta_{2}^{2}\right)
$$

This leads to

$$
\Delta \mathcal{L}=\left[-\frac{M_{1}}{4} \lambda \lambda-\frac{M_{2}}{4} \psi \psi-\left(\frac{M_{1} M_{2}}{4}-\frac{M_{3}}{4}\right) \phi \phi+h . c .\right]-\left(\frac{M_{1}^{2}}{4}+\frac{M_{2}^{2}}{4}\right) \bar{\phi} \phi,
$$

where the fields $\lambda$ are the gauginos, $\psi$ and $\phi$ are the spinor and scalar matter fields, respectively.

Now one can use the power of duality in N=2 SUSY theory and take the Seiberg-Witten solution 42

$$
\tau=\frac{d a_{D}}{d u} / \frac{d a}{d u}
$$

where

$$
a_{D}(u)=\frac{i}{2}(u-1) F\left(\frac{1}{2}, \frac{1}{2}, 2 ; \frac{1-u}{2}\right), a(u)=\sqrt{2(1+u)} F\left(-\frac{1}{2}, \frac{1}{2}, 1 ; \frac{2}{1+u}\right) .
$$

In perturbative domain when $u \sim Q^{2} / \Lambda^{2} \rightarrow \infty, a=\sqrt{2 u}, \quad a_{D}=\frac{i}{\pi} a(2 \ln a+1)$ one reproduces the well known one-loop result

$$
\frac{4 \pi}{g^{2}}=\frac{1}{\pi}\left[\ln \frac{Q^{2}}{\Lambda^{2}}+3\right]
$$

Assuming that renormalizations in $\mathrm{N}=2 \mathrm{SUSY}$ theory follow the properties of those in $\mathrm{N}=1$, one can try to apply the same expansion procedure for a non-perturbative solution. Substituting eq.(79) into (80) with

$$
u \rightarrow \tilde{u}=u\left(1+M_{1}^{0} \theta_{1}^{2}+M_{2}^{0} \theta_{2}^{2}+M_{3}^{0} \theta_{1}^{2} \theta_{2}^{2}\right)
$$


and expanding over $\theta_{1}^{2}$ and $\theta_{2}^{2}$, one gets an analog of S-W solution for the mass terms 43:

$$
\begin{gathered}
M_{1}=M_{1}^{0} \frac{\mathcal{I} m\left[u\left(\frac{a_{D}^{\prime \prime}}{a_{D}^{\prime}}-\frac{a^{\prime \prime}}{a^{\prime}}\right) \tau\right]}{\mathcal{I} m \tau}, \quad M_{2}=M_{2}^{0} \frac{\mathcal{I} m\left[u\left(\frac{a_{D}^{\prime \prime}}{a_{D}^{\prime}}-\frac{a^{\prime \prime}}{a^{\prime}}\right) \tau\right]}{\mathcal{I} m \tau}, \\
M_{3}=\frac{\mathcal{I} m\left[M_{3}^{0} u\left(\frac{a_{D}^{\prime \prime}}{a_{D}^{\prime}}-\frac{a^{\prime \prime}}{a^{\prime}}\right) \tau+M_{1}^{0} M_{2}^{0} u^{2}\left(\frac{a_{D}^{\prime \prime \prime}}{a_{D}^{\prime}}-\frac{a^{\prime \prime \prime}}{a^{\prime}}+2 \frac{a_{D}^{\prime \prime}}{a_{D}^{\prime}}\left(\frac{a_{D}^{\prime \prime}}{a_{D}^{\prime}}-\frac{a^{\prime \prime}}{a^{\prime}}\right)\right) \tau\right]}{\mathcal{I} m \tau}
\end{gathered}
$$

In perturbative regime one has

$$
M_{1}=\frac{M_{1}^{0}}{\ln Q^{2} / \Lambda^{2}+3}, \quad M_{2}=\frac{M_{2}^{0}}{\ln Q^{2} / \Lambda^{2}+3}, \quad M_{3}=\frac{M_{3}^{0}-M_{1}^{0} M_{2}^{0}}{\ln Q^{2} / \Lambda^{2}+3}
$$

\section{Renormalization of the Fayet-Iliopoulos Term}

We gave above a complete set of the rules needed for writing down the RG equations for the soft SUSY breaking terms in an arbitrary non-Abelian N=1 SUSY gauge theory. However, in the Abelian case, there exists an additional gauge invariant term, the so-called Fayet-Iliopoulos or the D-term [44]

$$
\mathcal{L}_{F . I .}=\xi D=\int d^{4} \theta \xi V
$$

which requires special consideration. In Ref. [45], it has been shown that in the unbroken theory this term is not renormalized provided the sum of hypercharges and their cubes equals zero. These requirements guarantee the absence of chiral and gravity anomalies and are usually satisfied in realistic models.

In case of a softly broken Abelian SUSY gauge theory, the F-I term happens to be renormalized even if anomalies are cancelled. The RG equation for $\xi$ depends not only on itself, but on the other soft breaking parameters (the soft mass of chiral scalars $m^{2}$, the soft triple coupling $A^{i j k}$ and the gaugino masses $M_{i}$ ). Recently, the renormalization of $\xi$ has been performed up to three loops [46] using the component approach and/or superfields with softly broken Feynman rules. Following our main idea that renormalizations of a softly broken SUSY theory are completely defined by a rigid one, we argue that the renormalization of the F-I term, in full analogy with all the other soft terms renormalizations, is completely defined in a rigid or an unbroken theory. However, contrary to the other soft renormalizations, there is no simple differential operator that acts on the renormalization functions of a rigid theory and allows one to get the renormalization of the F-I term. One needs an analysis of the superfield diagrams and some additional diagram calculations in components.

The addition of the F-I term leads to the modification of the Lagrangian in components. The relevant part of the Lagrangian is

$$
\mathcal{L}=\frac{1}{2 g^{2}} D^{2}+\xi D+D \bar{\phi}^{j} \mathcal{Y}_{j}^{i} \phi_{i}-\bar{\phi}^{j}\left(m^{2}\right)_{j}^{i} \phi_{i}+\ldots
$$


where $\mathcal{Y}_{j}^{i}$ is the hypercharge matrix of the chiral supermultiplet, and $\left(m^{2}\right)_{j}^{i}$ is a soft scalar mass. After eliminating the auxiliary field $D$ this becomes

$$
\mathcal{L}=-\bar{\phi}^{j}\left(m^{2}\right)_{j}^{i} \phi_{i}-\frac{1}{2} g^{2}\left(\bar{\phi}^{j} \mathcal{Y}_{j}^{i} \phi_{i}\right)^{2}+\ldots
$$

where

$$
\left(m^{2}\right)_{j}^{i}=\left(m^{2}\right)_{j}^{i}+g^{2} \xi \mathcal{Y}_{j}^{i}
$$

From eqs. 84 ) and (85) it follows that the F-I term gives an additional contribution to the renormalization of the soft scalar mass $\left(m^{2}\right)_{j}^{i}$

$$
\left[\beta_{m^{2}}\right]_{j}^{i}=\left[\beta_{m^{2}}\right]_{j}^{i}+\beta_{g^{2}} \xi \mathcal{Y}_{j}^{i}+g^{2} \beta_{\xi}\left(m^{2}, \ldots\right) \mathcal{Y}_{j}^{i}=\left[\beta_{m^{2}}\right]_{j}^{i}+g^{2} \beta_{\xi}\left(m^{2}, \ldots\right) \mathcal{Y}_{j}^{i} .
$$

The last equality follows from the fact that eq.(84) does not contain $\xi$ explicitly and, hence, $\xi$ should be dropped from all the expressions.

There are four different types of contributions to the renormalization of the F-I term in a softly broken theory: those proportional to $\left(m^{2}\right)_{j}^{i}, M \bar{M}, A^{i j k} \bar{A}_{l m n}$ and $M \bar{A}_{l m n}\left(\bar{M} A^{i j k}\right)$.

We have found that all the information about the renormalizations of the soft SUSY breaking terms is contained in a rigid, unbroken theory. To calculate the renormalization of an additional Fayet-Iliopoulos term, one needs an analysis of superfield diagrams. To find the contribution proportional to the soft scalar mass $\left(m^{2}\right)_{j}^{i}$ (the square of gaugino mass $M \bar{M})$, one needs to take the self-energy diagrams for the vector superfield and replace one of the external vertices with the hypercharge $\mathcal{Y}_{j}^{i}$ by $\left(m^{2}\right)_{j}^{i}\left(M \bar{M} \delta_{j}^{i}\right)$. In this case, there is no need to do any calculations except in superfields.

The other contributions (proportional to $A \bar{A}$ and $M \bar{A}$ ) can be found from the analysis of the matter superfield propagator diagrams in a rigid theory and the corresponding component diagrams in a softly broken theory extracting from the latter the contribution of the tadpole graphs. In this case, one needs to calculate additionally some component diagrams the number of which is essentially reduced compared to a direct component calculation [5].

\section{Conclusion}

Summarizing, we would like to stress once again that is very useful to consider a spontaneously broken theory in terms of a rigid one in an external field. In case when one is able to absorb the external field into the redefinition of parameters of the original theory and perform the renormalizations for an arbitrary field, one can reproduce renormalization properties of a spontaneously broken theory from a rigid one. The Grassmannian expansion in softly broken SUSY theories happens to be a very efficient and powerful method which can be applied in various cases where the renormalization procedure in concerned. It demonstrates once again that softly broken SUSY theories are contained in rigid ones and inherit their renormalization properties.

The following statements are valid:

- All the renormalizations are defined in a rigid theory. There are no independent renormalizations in a softly broken theory.

- RG flow in a softly broken theory follows that in a rigid theory. 
- This statement is true for RG equations, solutions to these equations, particular (fixed point) solutions, approximate solutions, etc.

- Renormalization of the F-I term needs a special treatment but can be also deduced from unbroken theory.

\section{Appendix A. Three-loop renormalizations in the MSSM}

In this section, we present explicit formulae for rigid and soft term renormalizations in the MSSM in the three-loop approximation in the case when we retain only $\alpha_{3}$ and top Yukawa coupling $Y_{t}$.

The rigid renormalizations are [16]

$$
\begin{aligned}
\beta_{\alpha_{3}} & =-3 \alpha_{3}^{2}+\alpha_{3}^{2}\left(14 \alpha_{3}-4 Y_{t}\right)+\alpha_{3}^{2}\left[\frac{347}{3} \alpha_{3}^{2}-\frac{104}{3} \alpha_{3} Y_{t}+30 Y_{t}^{2}\right] \\
\gamma_{t} & =\left(2 Y_{t}-\frac{8}{3} \alpha_{3}\right)-\left(8 Y_{t}^{2}+\frac{8}{9} \alpha_{3}^{2}\right)+\left[\left(30+12 \zeta_{3}\right) Y_{t}^{3}\right. \\
& \left.+\left(\frac{16}{3}+96 \zeta_{3}\right) Y_{t}^{2} \alpha_{3}-\left(\frac{64}{3}+\frac{544}{3} \zeta_{3}\right) Y_{t} \alpha_{3}^{2}+\left(\frac{2720}{27}+320 \zeta_{3}\right) \alpha_{3}^{3}\right] \\
\gamma_{b} & =-\frac{8}{3} \alpha_{3}-\frac{8}{9} \alpha_{3}^{2}+\left[-\frac{80}{3} Y_{t} \alpha_{3}^{2}+\left(\frac{2720}{27}+320 \zeta_{3}\right) \alpha_{3}^{3}\right] \\
\gamma_{Q} & =\left(Y_{t}-\frac{8}{3} \alpha_{3}\right)-\left(5 Y_{t}^{2}+\frac{8}{9} \alpha_{3}^{2}\right)+\left[\left(15+6 \zeta_{3}\right) Y_{t}^{3}\right. \\
& \left.+\left(\frac{40}{3}+48 \zeta_{3}\right) Y_{t}^{2} \alpha_{3}-\left(\frac{72}{3}+\frac{272}{3} \zeta_{3}\right) Y_{t} \alpha_{3}^{2}+\left(\frac{2720}{27}+320 \zeta_{3}\right) \alpha_{3}^{3}\right] \\
\gamma_{H_{2}} & =\left(3 Y_{t}\right)-\left(9 Y_{t}^{2}-16 Y_{t} \alpha_{3}\right) \\
& +\left[\left(57+18 \zeta_{3}\right) Y_{t}^{3}+\left(72-144 \zeta_{3}\right) Y_{t}^{2} \alpha_{3}-\left(\frac{160}{3}+16 \zeta_{3}\right) Y_{t} \alpha_{3}^{2}\right] \\
\beta_{Y_{t}} & =Y_{t}\left\{\left(6 Y_{t}-\frac{16}{3} \alpha_{3}\right)-\left(22 Y_{t}^{2}-16 Y_{t} \alpha_{3}+\frac{16}{9} \alpha_{3}^{2}\right)+\left[\left(102+36 \zeta_{3}\right) Y_{t}^{3}\right.\right. \\
& \left.\left.+\frac{272}{3} Y_{t}^{2} \alpha_{3}-\left(\frac{296}{3}+288 \zeta_{3}\right) Y_{t} \alpha_{3}^{2}+\left(\frac{5440}{27}+640 \zeta_{3}\right) \alpha_{3}^{3}\right]\right\} \\
\beta_{\mu^{2}} & =\mu^{2}\left\{3 Y_{t}-\left(9 Y_{t}^{2}-16 Y_{t} \alpha_{3}\right)\right. \\
+ & {\left.\left[\left(57+18 \zeta_{3}\right) Y_{t}^{3}+\left(72-144 \zeta_{3}\right) Y_{t}^{2} \alpha_{3}-\left(\frac{160}{3}+16 \zeta_{3}\right) Y_{t} \alpha_{3}^{2}\right]\right\} }
\end{aligned}
$$

Using the explicit form of anomalous dimensions calculated up to some order, one can reproduce the desired $\mathrm{RG}$ equations for the soft terms. In case of squark and slepton masses, they contain the contributions from unphysical masses $\Sigma_{\alpha_{i}}$. To eliminate them, one has to solve the equation for $\Sigma_{\alpha_{i}}$. In the case of the MSSM up to three loops, the solutions are 4

$$
\begin{aligned}
\Sigma_{\alpha_{1}} & =M_{1}^{2}-\alpha_{1} \sigma_{1}-\frac{199}{25} \alpha_{1}^{2} M_{1}^{2}-\frac{27}{5} \alpha_{1} \alpha_{2} M_{2}^{2}-\frac{88}{5} \alpha_{1} \alpha_{3} M_{3}^{2} \\
& +\frac{13}{5} \alpha_{1} Y_{t}\left(\Sigma_{t}+A_{t}^{2}\right)+\frac{7}{5} \alpha_{1} Y_{b}\left(\Sigma_{b}+A_{b}^{2}\right)+\frac{9}{5} \alpha_{1} Y_{\tau}\left(\Sigma_{\tau}+A_{\tau}^{2}\right) \\
\Sigma_{\alpha_{2}} & =M_{2}^{2}-\alpha_{2}\left(\sigma_{2}-4 M_{2}^{2}\right)-\alpha_{2}^{2}\left(4 \sigma_{2}+9 M_{2}^{2}\right)-\frac{9}{5} \alpha_{2} \alpha_{1} M_{1}^{2}-24 \alpha_{2} \alpha_{3} M_{3}^{2}
\end{aligned}
$$




$$
\begin{aligned}
& +3 \alpha_{2} Y_{t}\left(\Sigma_{t}+A_{t}^{2}\right)+3 \alpha_{2} Y_{b}\left(\Sigma_{b}+A_{b}^{2}\right)+\alpha_{2} Y_{\tau}\left(\Sigma_{\tau}+A_{\tau}^{2}\right), \\
\Sigma_{\alpha_{3}} & =M_{3}^{2}-\alpha_{3}\left(\sigma_{3}-6 M_{3}^{2}\right)-\alpha_{3}^{2}\left(6 \sigma_{3}-22 M_{3}^{2}\right)-\frac{11}{5} \alpha_{3} \alpha_{1} M_{1}^{2}-9 \alpha_{3} \alpha_{2} M_{2}^{2} \\
& +2 \alpha_{3} Y_{t}\left(\Sigma_{t}+A_{t}^{2}\right)+2 \alpha_{3} Y_{b}\left(\Sigma_{b}+A_{b}^{2}\right),
\end{aligned}
$$

where we have used the combinations 25]

$$
\begin{aligned}
\sigma_{1} & =\frac{1}{5}\left[3\left(m_{H_{1}}^{2}+m_{H_{2}}^{2}\right)+3\left(\tilde{m}_{Q}^{2}+3 \tilde{m}_{L}^{2}+8 \tilde{m}_{U}^{2}+2 \tilde{m}_{D}^{2}+6 \tilde{m}_{E}^{2}\right)\right] \\
\sigma_{2} & =m_{H_{1}}^{2}+m_{H_{2}}^{2}+3\left(3 \tilde{m}_{Q}^{2}+\tilde{m}_{L}^{2}\right) \\
\sigma_{3} & =3\left(2 \tilde{m}_{Q}^{2}+\tilde{m}_{U}^{2}+\tilde{m}_{D}^{2}\right), \\
\Sigma_{t} & =\tilde{m}_{t}^{2}+\tilde{m}_{Q}^{2}+m_{H_{2}}^{2}, \quad \Sigma_{b}=\tilde{m}_{b}^{2}+\tilde{m}_{Q}^{2}+m_{H_{1}}^{2}, \quad \Sigma_{\tau}=\tilde{m}_{\tau}^{2}+\tilde{m}_{L}^{2}+m_{H_{1}}^{2} .
\end{aligned}
$$

The corresponding soft term renormalizations read

$$
\begin{aligned}
& \beta_{M_{3}}=-3 \alpha_{3} M_{3}+28 \alpha_{3}^{2} M_{3}-4 Y_{t} \alpha_{3}\left(M_{3}-A_{t}\right) \\
& +347 \alpha_{3}^{3} M_{3}-\frac{104}{3} \alpha_{3}^{2} Y_{t}\left(2 M_{3}-A_{t}\right)+30 \alpha_{3} Y_{t}^{2}\left(M_{3}-2 A_{t}\right), \\
& \beta_{A_{t}}=\left(6 Y_{t} A_{t}+\frac{16}{3} \alpha_{3} M_{3}\right)-\left[44 Y_{t}^{2} A_{t}-16 Y_{t} \alpha_{3}\left(A_{t}-M_{3}\right)-\frac{32}{9} \alpha_{3}^{2} M_{3}\right] \\
& +\left[\left(306+108 \zeta_{3}\right) Y_{t}^{3} A_{t}+\frac{272}{3} Y_{t}^{2} \alpha_{3}\left(2 A_{t}-M_{3}\right)\right. \\
& \text { - } \left.\left(\frac{296}{3}+288 \zeta_{3}\right) Y_{t} \alpha_{3}^{2}\left(A_{t}-2 M_{3}\right)-3\left(\frac{5440}{27}+640 \zeta_{3}\right) \alpha_{3}^{3} M_{3}\right] \text {, } \\
& \beta_{B}=3 Y_{t} A_{t}-\left[18 Y_{t}^{2} A_{t}-16 Y_{t} \alpha_{3}\left(A_{t}-M_{3}\right)\right]+\left[\left(171+54 \zeta_{3}\right) Y_{t}^{3} A_{t}\right. \\
& \left.+\left(72-144 \zeta_{3}\right) Y_{t}^{2} \alpha_{3}\left(2 A_{t}-M_{3}\right)-\left(\frac{160}{3}+16 \zeta_{3}\right) Y_{t} \alpha_{3}^{2}\left(A_{t}-2 M_{3}\right)\right] \text {, } \\
& \beta_{\tilde{m}_{t}^{2}}=2 Y_{t}\left(\Sigma_{t}+A_{t}^{2}\right)-\frac{16}{3} \alpha_{3} M_{3}^{2}-16 Y_{t}^{2}\left(\Sigma_{t}+2 A_{t}^{2}\right)-\frac{64}{3} \alpha_{3}^{2} M_{3}^{2}+\frac{8}{3} \alpha_{3}^{2} \sigma_{3} \\
& +3\left(30+12 \zeta_{3}\right) Y_{t}^{3}\left(\Sigma_{t}+3 A_{t}^{2}\right)+\left(\frac{16}{3}+96 \zeta_{3}\right) Y_{t}^{2} \alpha_{3}\left[\left(2 A_{t}-M_{3}\right)^{2}\right. \\
& \left.+2 \Sigma_{t}+M_{3}^{2}\right]-\left(\frac{64}{3}+\frac{544}{3} \zeta_{3}\right) Y_{t} \alpha_{3}^{2}\left[\left(A_{t}-2 M_{3}\right)^{2}+\Sigma_{t}+2 M_{3}^{2}\right] \\
& -\frac{16}{3} Y_{t} \alpha_{3}^{2}\left(\Sigma_{t}+A_{t}^{2}\right)+4\left(\frac{2564}{9}+960 \zeta_{3}\right) \alpha_{3}^{3} M_{3}^{2}+\frac{160}{9} \alpha_{3}^{3} \sigma_{3} \text {, } \\
& \beta_{\tilde{m}_{b}^{2}}=-\frac{16}{3} \alpha_{3} M_{3}^{2}-\frac{64}{3} \alpha_{3}^{2} M_{3}^{2}+\frac{8}{3} \alpha_{3}^{2} \sigma_{3}-\frac{80}{3} Y_{t} \alpha_{3}^{2}\left[\left(A_{t}-2 M_{3}\right)^{2}+\Sigma_{t}\right. \\
& \left.+2 M_{3}^{2}\right]-\frac{16}{3} Y_{t} \alpha_{3}^{2}\left(\Sigma_{t}+A_{t}^{2}\right)+4\left(\frac{2564}{9}+960 \zeta_{3}\right) \alpha_{3}^{3} M_{3}^{2}+\frac{160}{9} \alpha_{3}^{3} \sigma_{3}, \\
& \beta_{\tilde{m}_{Q}^{2}}=Y_{t}\left(\Sigma_{t}+A_{t}^{2}\right)-\frac{16}{3} \alpha_{3} M_{3}^{2}-10 Y_{t}^{2}\left(\Sigma_{t}+2 A_{t}^{2}\right)-\frac{64}{3} \alpha_{3}^{2} M_{3}^{2}+\frac{8}{3} \alpha_{3}^{2} \sigma_{3} \\
& +3\left(15+6 \zeta_{3}\right) Y_{t}^{3}\left(\Sigma_{t}+3 A_{t}^{2}\right)+\left(\frac{40}{3}+48 \zeta_{3}\right) Y_{t}^{2} \alpha_{3}\left[\left(2 A_{t}-M_{3}\right)^{2}\right. \\
& \left.+2 \Sigma_{t}+M_{3}^{2}\right]-\left(\frac{72}{3}+\frac{272}{3} \zeta_{3}\right) Y_{t} \alpha_{3}^{2}\left[\left(A_{t}-2 M_{3}\right)^{2}+\Sigma_{t}+2 M_{3}^{2}\right] \\
& -\frac{16}{3} Y_{t} \alpha_{3}^{2}\left(\Sigma_{t}+A_{t}^{2}\right)+4\left(\frac{2564}{9}+960 \zeta_{3}\right) \alpha_{3}^{3} M_{3}^{2}+\frac{160}{9} \alpha_{3}^{3} \sigma_{3}, \\
& \beta_{m_{H_{2}}^{2}}=3 Y_{t}\left(\Sigma_{t}+A_{t}^{2}\right)-18 Y_{t}^{2}\left(\Sigma_{t}+2 A_{t}^{2}\right)+16 Y_{t} \alpha_{3}\left[\left(A_{t}-M_{3}\right)^{2}+\Sigma_{t}+M_{3}^{2}\right]
\end{aligned}
$$




$$
\begin{aligned}
& +3\left(57+18 \zeta_{3}\right) Y_{t}^{3}\left(\Sigma_{t}+3 A_{t}^{2}\right)+\left(72-144 \zeta_{3}\right) Y_{t}^{2} \alpha_{3}\left[\left(2 A_{t}-M_{3}\right)^{2}\right. \\
& \left.+\quad 2 \Sigma_{t}+M_{3}^{2}\right]-\left(\frac{160}{3}+16 \zeta_{3}\right) Y_{t} \alpha_{3}^{2}\left[\left(A_{t}-2 M_{3}\right)^{2}+\Sigma_{t}+2 M_{3}^{2}\right] \\
& -16 Y_{t} \alpha_{3}^{2}\left(\sigma_{3}-6 M_{3}^{2}\right) .
\end{aligned}
$$

\section{Appendix B}

The RG equation for the parameter $\xi$ in a rigid theory is

$$
\dot{\xi}=-\gamma_{V} \xi
$$

where $\gamma_{V}$ is the anomalous dimension of the gauge superfield. To find the soft terms $x, \bar{x}$ and $z$, one should solve the modified equation

$$
\dot{\tilde{\xi}}=-\gamma_{V}(\tilde{\alpha}, \tilde{y}, \tilde{\xi}) \tilde{\xi}
$$

In one-loop order $\gamma_{V}=\left(b_{1}+b_{2} \xi\right) \alpha$, where $b_{1}+b_{2}=Q$, and the solutions are

$$
\begin{aligned}
& x=-\left(M+x_{0}\right) \frac{b_{1}+b_{2} \xi}{Q}, \quad \bar{x}=-\left(\bar{M}+\bar{x}_{0}\right) \frac{b_{1}+b_{2} \xi}{Q}, \\
& z=-\left(\Sigma_{\alpha}+z_{0}\right) \frac{b_{1}+b_{2} \xi}{Q}+\frac{b_{2} \xi}{Q}\left(M+x_{0}\right)\left(\bar{M}+\bar{x}_{0}\right) \frac{b_{1}+b_{2} \xi}{Q},
\end{aligned}
$$

where $x_{0}, \bar{x}_{0}$, and $z_{0}$ are arbitrary constants. In the Abelian case when $b_{1}=Q, b_{2}=0$, the solutions are simplified and can be chosen as

$$
x=-M(1-\xi), \quad \bar{x}=-\bar{M}(1-\xi), \quad z=-\Sigma_{\alpha}(1-\xi)-M \bar{M} \xi(1-\xi) .
$$

Together with the expression for $\tilde{\alpha}$ (14) it gives eq.(19) above.

\section{Acknowledgments}

I would like to thank my colleagues L.Avdeev, I.Kondrashuk, S.Codoban, G.Moultaka and V.Velizhanin in collaboration with whom these results have been obtained. I am grateful to the organizers of the conference "Continuous Advances in QCD-02" and especially to M.Shifman for their invitation to participate in the conference and to present this report. Many thanks are to M.Shifman, A.Vainshtein, M.Strassler, I.Jack, T.Jones and R.Rattazzi for useful discussions.

Financial support from RFBR grants \# 02-02-16889 and \# 00-15-96691 is kindly acknowledged.

\section{References}

[1] L.A.Avdeev, D.I.Kazakov and I.N.Kondrashuk, Nucl.Phys. B510 (1998) 289.

[2] D. I. Kazakov, Phys.Lett. B421 (1998) 211.

[3] D. I. Kazakov, Phys.Lett. B449 (1999) 201. 
[4] D. I. Kazakov and V. N. Velizhanin, Phys.Lett. B485 (2000) 393.

[5] D. I. Kazakov and V. N. Velizhanin, Phys.Rev. D65 (2002) 085041.

[6] I. Jack and D.R.T. Jones, Phys.Lett. B415 (1997) 383.

[7] G. F. Giudice and R. Rattazzi, Nucl.Phys. B511 (1998) 25.

[8] A. Nelson and M. Strassler, JHEP 0207 (2002) 021.

[9] Y. Yamada, Phys.Rev. D50 (1994) 3537.

[10] I. Jack, D.R.T. Jones and A. Pickering, Phys.Lett. B426 (1998) 73.

[11] A. A. Slavnov and K. V. Stepanyantz, hep-th/0208006; V. K. Krivoshchekov, Theor.Math.Phys. 36 (1978) 291.

[12] L. V. Avdeev, G. A. Chochia and A. A. Vladimirov, Phys.Lett. B105 (1981) 272.

[13] M. Vaughn and S. Martin, Phys. Lett. B318 (1993) 331.

[14] I. Jack and D.R.T. Jones, Phys. Lett. B333 (1994) 372.

[15] I. Jack, D.R.T. Jones and C.G. North, Phys.Lett. B386 (1996) 138.

[16] P.M. Ferreira, I. Jack and D.R.T. Jones, Phys.Lett. B387 (1996) 80.

[17] S. Ferrara and O. Piguet, Nucl.Phys. B93 (1975) 261.

[18] L. Girardello and M.T. Grisaru, Nucl.Phys. B194 (1982) 65.

[19] I. Jack and D. R. T. Jones, Phys. Lett. B457 (1999) 101.

[20] M. A. Shifman, A. I. Vainshtein and V. I. Zakharov, Yad.Fiz. 43 (1986) 1596; M. A. Shifman and A. I. Vainshtein Nucl.Phys. B359 (1991) 571.

[21] I. N. Kondrashuk, J.Phys. A33 (2000) 6399.

[22] W. Siegel, Phys.Lett. B84 (1979) 193; R. van Damme and G. 't Hooft, Phys. Lett. B150 (1985) 133.

[23] I. Jack and D. R. T. Jones, Phys. Lett. B415 (1997) 383.

[24] I. Jack, D. R. T. Jones, S. P. Martin, M. T. Voughn and Y. Yamada, Phys.Rev. D50 (1994) 5481.

[25] S. P. Martin and M. T. Voughn, Phys.Rev. D50 (1994) 2282.

[26] I. Jack and D. R. T. Jones, Phys. Lett. B333 (1994) 372.

[27] J.A. Helayël-Neto, Phys.Lett. 135B (1984) 78; F. Feruglio, J.A. Helayël-Neto and F. Legovini, Nucl. Phys. B249 (1985) 533; M. Scholl, Z. Phys. C28 (1985) 545.

[28] J. Hisano and M. A. Shifman, Phys.Rev. D56 (1997) 5475. 
[29] T. Kobayashi and K. Yoshioka, Phys. Lett. B486 (2000)223.

[30] I. Jack, D. R. T. Jones and A. Pickering, Phys.Lett. B432 (1998) 114.

[31] V. A. Novikov, M. A. Shifman, A. I. Vainshtein and V. I. Zakharov, Nucl. Phys. B229 (1983) 381.

[32] T. Kobayashi, J. Kubo and G. Zoupanos, Phys.Lett. B427 (1998) 291.

[33] L.E. Ibáñez, C. López and C. Muñoz, Nucl.Phys. B256 (1985) 218.

[34] C. T. Hill, Phys.Rev. D24 (1981) 691; C. T. Hill, C.N. Leung and S. Rao, Nucl. Phys. B262 (1985) 517.

[35] I. Jack and D. R. T. Jones, Phys. Lett. B443 (1998) 177.

[36] G. Auberson and G. Moultaka, Eur.Phys.J. C12 (2000) 331.

[37] S. Codoban and D. I. Kazakov, Eur.Phys.J C13 (2000) 671.

[38] D. I. Kazakov and G. Moultaka, Nucl.Phys. B577 (2000) 121.

[39] P. C. West, Phys.Lett. 137B (1984) 371; A. J. Parkes and P. C. West, Phys.Lett. 138B (1984) 99; D.R.T. Jones and L. Mezinchescu, Phys.Lett. 138B (1984) 293, S. Hamidi, J. Patera and J. H. Schwarz, Phys.Lett. 141B (1984) 349.

[40] A. V. Ermushev, D. I. Kazakov and O. V. Tarasov, Nucl.Phys. B281 (1987) 72; D.R.T. Jones, Nucl.Phys. B277 (1986) 153; C. Lucchesi, O. Piguet and K. Sibold, Phys.Lett. 201B (1988) 241; D. I. Kazakov, Mod.Phys.Lett. A9 (1987) 663.

[41] see e.g. L. Alvarez-Gaume and S.F. Hassan, Fortsch.Phys. 45 (1997) 159.

[42] N. Seiberg and E. Witten, Nucl.Phys. B426 (1994) 19.

[43] D. I. Kazakov and V. N. Velizhanin, Part $\mathscr{E}$ Nucl. 31 (2000) 240. Proc. of Bogoliubov Conf., Dubna, 1999.

[44] P. Fayet and J. Iliopoulos, Phys.Lett. B51 (1974) 461.

[45] W. Fischler, H. P. Nilles, J. Polchinski, S. Raby and L. Susskind, Phys.Rev.Lett. 47 (1981) 757.

[46] I. Jack, D. R. T. Jones and S. Parsons, Phys.Rev. D62 (2000) 125022. 